\title{
CONNECTION MATRICES FOR MORSE-BOTT FLOWS
}

\author{
Dahisy V. de S. Lima - Ketty A. de Rezende
}

\begin{abstract}
A Connection Matrix Theory approach is presented for MorseBott flows $\varphi$ on smooth closed $n$-manifolds by characterizing the set of connection matrices in terms of Morse-Smale perturbations. Further results are obtained on the effect on the set of connection matrices $\mathcal{C M}(S)$ caused by changes in the partial orderings and in the Morse decompositions of an isolated invariant set $S$.
\end{abstract}

\section{Introduction}

Our goal is to make use of Conley Index Theory, [3], to study Morse-Bott flows on a smooth closed $n$-manifold $M$ with the underlying motivation of obtaining dynamical information from homotopical invariants.

In [9], the Conley index of critical manifolds of Morse-Bott functions were thoroughly investigated and the generalized Morse-Bott inequalities were introduced, bearing in mind continuation results of graphs. In this article, we wish to introduce a Connection Matrix Theory approach for Morse-Bott flows. The motivation for this resides in the fact that connection matrices, as defined by Franzosa in [5], [6] and [7], were introduced to study the behaviour of connecting orbits in a flow which undergoes perturbation. Eventual bifurcations were captured by transition matrices, see also [8], [10] and [12]. On the other hand, in

2010 Mathematics Subject Classification. 37B30, 57R70.

Key words and phrases. Morse decompositions, Conley index, connection matrices, MorseBott functions, Morse functions.

The first named author supported by FAPESP under grant 2010/08579-0.

The second named author partially supported by CNPq under grant 302592/2010-5 and by FAPESP under grant 2012/18780-0. 
the setting of Morse Theory, connection matrices can be viewed as differentials of Morse complexes (see [14]), making it possible to translate topological data into dynamical data and vice versa.

Considering a Morse-Bott function $f: M \rightarrow \mathbb{R}$ on a smooth closed $n$-manifold $M$, a Morse-Bott complex associated to $f$ is constructed in [1] by means of a Morse-Smale perturbation $h: M \rightarrow \mathbb{R}$ of $f$. A natural question to consider is whether the differential of a Morse-Bott complex can be interpreted as a connection matrix. The answer to this is affirmative. However, a necessary step to accomplish this endeavour is to explore more deeply the Connection Matrix Theory for Morse-Bott flows, which is our focus in this paper.

The idea is to obtain a characterization of the set of connection matrices for a Morse-Bott flow $\varphi_{f}$ on $M$ using the set of connection matrices for a MorseSmale flow $\varphi_{h}$ on $M$, where $h$ is a Morse perturbation of $f$ and $\varphi_{f}$ (resp. $\varphi_{h}$ ) is a flow associated to the vector field $-\nabla f$ (resp. $-\nabla h)$. As a result of this characterization, proved in Section 4, one can define a Morse-Bott complex with differential being a connection matrix which opens the possibility to the use of spectral sequence techniques, such as in [4], [11] and [8], to obtain further dynamical information, e.g. bifurcating orbits.

Our approach to study connection matrices for Morse-Bott flows and obtain the required characterization is to initially consider connection matrices of Morse decompositions in a general setting. Given an isolated invariant set $S$, we analyze what properties remain on the sets of connection matrices when both Morse decompositions of $S$ and partial orderings undergo changes.

The article is organized as follows. In Section 2 we review some classical definitions and results on Morse decompositions and connection matrices and how these concepts are related to Morse chain complexes.

In Section 3 we consider connection matrices in a general framework. More specifically, given an isolated invariant set $S$ and a <-ordered Morse decomposition $\mathcal{D}(S)$, we measure the effect a change in the Morse decomposition $\mathcal{D}(S)$, caused by a modification on the partial order $<$, has on the respective connection matrices, as shown in Proposition 3.3 and Theorem 3.7.

In Section 4 we apply the results obtained in Section 3 to Morse-Bott flows $\varphi_{f}$ on a closed $n$-manifold $M$. For instance, in Theorem 4.2 we prove that the set of connection matrices for Morse-Bott flows on $M$ coincides with the set of connection matrices for perturbations that give rise to Morse-Smale flows on $M$. Theorem 4.3 is more constructive in nature, since we show how a connection matrix of $\mathcal{D}\left(M, \varphi_{h}\right)$, where $\varphi_{h}$ is a perturbation of $\varphi_{f}$, induces a connection matrix of the Morse-Bott flow $\varphi_{f}$.

In Section 5, we answer a natural question that arises in this context, which examines if for a given connection matrix $\Delta$ for a Morse-Bott flow $\varphi_{f}$ there exists 
a Morse-Smale perturbation $\varphi_{h}$ of $\varphi_{f}$ such that $\Delta$ is induced from a connection matrix of $\varphi_{h}$.

\section{Background}

We assume that the reader is familiar with the basic ideas in Conley Index Theory, including homotopical and homological Conley index, Morse decompositions, homology index braids, connection matrices, etc. Some references for this section are [3], [5], [6], [7] and [14].

2.1. Connection matrix theory. In this subsection, we present some definitions and results on connection matrices, Morse decompositions and partial orders. Further details can be found in [5] and [6], for example.

Let $P$ be a finite set. A partial order on $P$ is a transitive relation $<$ on the elements of $P$ for which $\pi<\pi$ never holds, for all $\pi \in P$. The pair $(P,<)$ is called a partially ordered set.

An interval in $(P,<)$ is a subset $I \subset P$, such that, if $\pi, \pi^{\prime} \in I$ and $\pi<\pi^{\prime \prime}<$ $\pi^{\prime}$, where $\pi^{\prime \prime} \in P$, then $\pi^{\prime \prime} \in I$.

The set of all intervals in $(P,<)$ is denoted by $\mathcal{I}(P,<)$. Two elements $\pi, \pi^{\prime}$ of $P$ are said to be adjacent if $\left\{\pi, \pi^{\prime}\right\} \in \mathcal{I}(P,<)$.

An ordered collection $\left(I_{1}, \ldots, I_{n}\right)$ of intervals in $(P,<)$ is called an adjacent $n$ tuple of intervals if $\bigcup_{j=1}^{n} I_{j} \in \mathcal{I}(P,<)$ and if $\pi \in I_{j}$ and $\pi^{\prime} \in I_{k}$, with $k<j$, implies $\pi \nless \pi^{\prime}$. The set of all adjacent $n$-tuples of intervals is denoted by $\mathcal{I}_{n}(P,<)$.

If $(I, J) \in \mathcal{I}_{2}(P,<)$ then $I \cup J$ is denoted by $I J$.

An $n$-tuple $\left(I_{1}, \ldots, I_{n}\right)$ is called a decomposition of an interval $I$ if $\left(I_{1}, \ldots, I_{n}\right)$ in $\mathcal{I}_{n}(P,<)$ and $\bigcup_{j=1}^{n} I_{j}=I$.

Let $\Gamma$ be a Hausdorff topological space with a continuous flow and $S$ an isolated invariant set in $\Gamma$. A <-ordered Morse decomposition of $S$ is a collection $\mathcal{D}(S)=\left\{M_{\pi}\right\}_{\pi \in P}$ of mutually disjoint compact invariant subsets of $S$ for which the following property holds: if $\gamma$ does not belong to any $M_{\pi}$ with $\pi \in P$, then there must exist $\pi, \pi^{\prime} \in P$ such that $\gamma \in C\left(M_{\pi}, M_{\pi^{\prime}}\right)$, where $C\left(M_{\pi}, M_{\pi^{\prime}}\right)$ is the set of orbits connecting $M_{\pi}$ to $M_{\pi^{\prime}}$, i.e.

$$
C\left(M_{\pi}, M_{\pi^{\prime}}\right)=\left\{x \in S \mid \omega^{*} \subset M_{\pi} \text { and } \omega \subset M_{\pi^{\prime}}\right\} .
$$

Note that the partial order $<$ on $P$ induces a partial order on $\mathcal{D}(S)$, which is also denoted by $<$ and called an admissible ordering of $\mathcal{D}(S)$. The flow defines an admissible ordering $<_{F}$ of $\mathcal{D}(S)$, defined as follows: $M_{\pi}<_{F} M_{\pi^{\prime}}$ if and only if there exists a sequence $\pi=\pi_{0}, \pi_{1}, \ldots, \pi_{n-1}, \pi_{n}=\pi^{\prime}$ of elements of $P$ such that $C\left(M_{\pi_{j}}, M_{\pi_{j-1}}\right) \neq \emptyset$, for all $j=1, \ldots n$. Every admissible ordering of $\mathcal{D}(S)$ is an extension of $<_{F}$, in other words, all other admissible orders are obtained by adding relations to $<_{F}$. 
For each interval $I$ of $(P,<)$, one can associate the set

$$
M_{I}=\left(\bigcup_{\pi \in I} M_{\pi}\right) \cup\left(\bigcup_{\pi, \pi \in I} C\left(M_{\pi^{\prime}}, M_{\pi}\right)\right),
$$

each is called a Morse set of the admissible ordering $<$. Franzosa proves in [5] that if $(I, J) \in \mathcal{I}_{2}(P,<)$ then $\left(M_{I}, M_{J}\right)$ is an attractor-repeller pair in $M_{I J}$ and that there is a long exact sequence

$$
\cdots \longrightarrow C H\left(M_{I}\right) \stackrel{i_{*}}{\longrightarrow} C H\left(M_{I J}\right) \stackrel{p_{*}}{\longrightarrow} C H\left(M_{J}\right) \stackrel{\partial_{*}}{\longrightarrow} C H\left(M_{I}\right) \longrightarrow \cdots
$$

associated to the pair $\left(M_{I}, M_{J}\right)$, where $C H\left(M_{I}\right)$ denotes de homology index of $M_{I}$. The collection of the homology index $C H\left(M_{I}\right)$, for all $I \in \mathcal{I}(P,<)$, and the maps $i_{*}, p_{*}, \partial_{*}$, for all pair $(I, J) \in \mathcal{I}_{2}(P,<)$, is a graded module braid over $<$. This graded module braid is denoted by $\mathcal{H}(<)$ and is called the homology index braid of the admissible ordering $<$ of $\mathcal{D}(S)$. Moreover, $\mathcal{H}(<)$ is chain complex generated. See [5] and [6] for more details.

Now, let $C=\{C \Delta(\pi)\}_{\pi \in P}$ be a collection of free chain complexes with trivial boundary operator, where $C \Delta(\pi)=C H\left(M_{\pi}\right)$, for all $\pi \in P$, the homology index of $M_{\pi}$ with coefficients in $G$. A map $\Delta: C \Delta(P) \rightarrow C \Delta(P)$ can be viewed as a matrix

$$
\Delta=\left(\Delta\left(\pi^{\prime}, \pi\right)\right)_{\pi, \pi^{\prime} \in P}
$$

where each entry $\Delta\left(\pi^{\prime}, \pi\right)$ is a map of degree -1 from $C H\left(M_{\pi^{\prime}}\right)$ to $C H\left(M_{\pi}\right)$. One says that

(a) $\Delta$ is strictly upper triangular if $\Delta\left(\pi^{\prime}, \pi\right) \neq 0$ implies $\pi<\pi^{\prime}$;

(b) $\Delta$ is a boundary map if each map $\Delta\left(\pi^{\prime}, \pi\right)$ is of degree -1 and $\Delta \circ \Delta=0$; Given $(I, J) \in \mathcal{I}_{2}(P,<)$, let $\Delta(J, I)$ be the map defined by the matrix

$$
\Delta(J, I)=\left(\Delta\left(\pi^{\prime}, \pi\right)\right)_{\pi \in I, \pi^{\prime} \in J}
$$

and denote the map $\Delta(I, I)$ by $\Delta(I)$. For each $I \in \mathcal{I}(P,<)$, considering the module $C \Delta(I)=\bigoplus_{\pi \in I} C H\left(M_{\pi}\right),(C \Delta(I), \Delta(I))$ is a chain complex. For each pair of adjacent intervals $(I, J)$, there is a short exact sequence associated to it:

$$
0 \longrightarrow C \Delta(I) \stackrel{i(I, I J)}{\longrightarrow} C \Delta(I J) \stackrel{p(I J, J)}{\longrightarrow} C \Delta(J) \longrightarrow 0 .
$$

Passing to homology, one has the long exact sequence

$$
\cdots \longrightarrow H \Delta(I) \stackrel{i_{*}(I, I J)}{\longrightarrow} H \Delta(I J) \stackrel{p_{*}(I J, J)}{\longrightarrow} H \Delta(J) \stackrel{\Delta_{*}(J, I)}{\longrightarrow} H \Delta(I) \longrightarrow \cdots,
$$

where $H \Delta(K)$ denotes the homology of the chain complex $(C \Delta(K), \Delta(K))$, the maps $i_{*}(I, I J), p_{*}(I J, J)$ are induced by the inclusion $i(I, I J)$ and projection $p(I J, J)$ maps, respectively. The map $\Delta_{*}(J, I)$ is induced by $\Delta(J, I)$ as follows: $\Delta_{*}(J, I)[a]=[\Delta(J, I) a]$. The collection of $H \Delta(I)$, for all $I \in \mathcal{I}(P,<)$, and the 
maps $i_{*}(I, I J), p_{*}(I J, J), \Delta_{*}(J, I)$, for each $(I, J) \in \mathcal{I}_{2}(P,<)$, is a graded module braid denoted by $\mathcal{H} \Delta$.

A strictly upper triangular boundary map $\Delta: C \Delta(P) \rightarrow C \Delta(P)$ is called a connection matrix of $\mathcal{H}(<)$ if and only if the graded module braid $\mathcal{H} \Delta$ generated by $\Delta$ is isomorphic to $\mathcal{H}(<)$, that is, if there is a collection of isomorphisms

$$
\left\{\theta(I): H \Delta(I) \rightarrow C H\left(M_{I}\right) \mid I \in \mathcal{I}(P,<)\right\},
$$

such that, the following diagram commutes for all pair $(I, J) \in \mathcal{I}_{2}(P,<)$ :

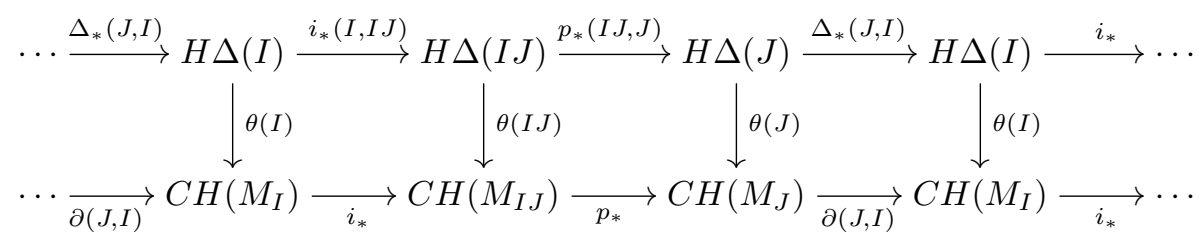

If $<$ is the flow ordering, then $\Delta$ is said to be a connection matrix of the Morse decomposition $\mathcal{D}(S)$.

The set $\mathcal{C M}(<)$ of connection matrices of $\mathcal{H}(<)$ is non empty, as Franzosa proved in [6]. Moreover, if $<_{1}$ and $<_{2}$ are admissible orderings of $\mathcal{D}(S)$ such that $<_{2}$ is an extension of $<_{1}$, then $\mathcal{C M}\left(<_{1}\right) \subset \mathcal{C M}\left(<_{2}\right)$. In particularly, $\mathcal{C M}\left(<_{F}\right) \subset$ $\mathcal{C M}(<)$, for all admissible ordering $<$ of $\mathcal{D}(S)$.

The set $\mathcal{C M}(<)$ provides some dynamical information about the structure of an invariant set $S$. A well known fact is that if $\Delta \in \mathcal{C M}\left(<_{F}\right), \pi$ and $\pi^{\prime}$ are adjacent in the flow ordering and $\Delta\left(\pi^{\prime}, \pi\right) \neq 0$ then $C\left(M_{\pi^{\prime}}, M_{\pi}\right) \neq \emptyset$.

Note that algebraic properties of $\Delta$ put restrictions on the maps $\partial\left(\pi, \pi^{\prime}\right)$. $\Delta$ can be used to prove the existence of connecting orbits between Morse sets. Moreover, this theory can also be applied to the study of parameterized families of flows, according to the following two approaches: first by studying the stability of connection matrices under perturbations, whenever some stable connecting orbits are identified; and secondly by studying the changes in connection matrices under perturbation, whenever bifurcations are detected, see [7] and [8].

2.2. Morse chain complex. Let $f$ be a Morse function on a smooth closed Riemannian manifold $M$ of finite dimension $n$. Denote by $\varphi_{f}$ a flow associated to the vector field $-\nabla f$. Assume that $f$ satisfies the transversality condition, i.e. the stable and unstable manifolds of critical points of $f$ have transversal intersection. In this case, we refer to $f$ as a Morse-Smale function. Given $x, y \in \operatorname{Crit}(f)$ of index $k$ and $k-1$, respectively, the set of orbits connecting $x$ to $y$ is finite. To count orbits with signs, fix orientations of the unstable manifold $W^{u}(x)$ of critical points $x$ of $f$, and denote by $\langle x\rangle$ both the critical point $x$ and the fixed orientation of $W^{u}(x)$. Now, define $n(x, y)$ as the intersection number between the spheres $S^{k-1}=W^{u}(x) \cap f^{-1}(a)$ and $S^{n-k}=W^{s}(y) \cap f^{-1}(a)$, where $a$ is a regular value between $f(x)$ and $f(y)$. For each $k=1, \ldots, n$, the $k$-th Morse 
chain group is

$$
C_{k}(f)=\bigoplus_{x \in \mathrm{Crit}_{k}(f)} \mathbb{Z}\langle x\rangle,
$$

the free abelian group generated by all critical points of $f$ of index $k$. Define the boundary operator $\partial_{k}^{c}: C_{k}(f) \rightarrow C_{k-1}(f)$ on the generators of $C_{k}(f)$ by

$$
\partial_{k}^{c}\langle x\rangle=\sum_{y \in \mathrm{Crit}_{k-1}(f)} n(x, y)\langle y\rangle,
$$

and extend it by linearity. The pair $\left(C_{*}(f), \partial_{*}^{c}\right)$ is the Morse chain complex over $\mathbb{Z}$. Analogously, one can define a Morse chain complex over $\mathbb{Z}_{2}$ by defining $n(x, y)$ as the number of orbits connecting $x$ to $y$ module 2 . For details about Morse chain complexes see [14] and [15].

A result due to Salamon, see [14], establishes a relation between the boundary operator $\partial_{*}^{c}$ of a Morse chain complex with connection matrices. Consider $M$ as an isolated invariant set relative to the flow $\varphi_{f}$ and the finest Morse decomposition $\mathcal{D}(M)=\left\{M_{x}=\{x\} \mid x \in \operatorname{Crit}(f)\right\}$ of $M$, i.e. each Morse set $M_{\pi}$ contains only one singularity of $\varphi_{f}$. Salamon proved in [14] that the boundary operator $\partial_{*}^{c}$ of a Morse chain complex associated to $f$ is a connection matrix of the Morse decomposition $\mathcal{D}(M)$, by identifying the free abelian group $\mathbb{Z}\langle x\rangle$ with the homology index $C H\left(M_{x}\right)$ of $M_{x}$. For more details, see [2] and [14].

In this setting of flows generated by Morse functions $f: M \rightarrow \mathbb{R}$, it is interesting to note that each connection matrix of the finest Morse decomposition of $M, \mathcal{D}(M)$, encodes the weak Morse inequalities, as mentioned in [6]. Indeed, denote the rank of $H_{k}(M ; \mathbb{Z})\left(^{1}\right)$, i.e. the $k$-th Betti number of $M$, by $\beta_{k}$ and the cardinality of the set of all critical points of $f$ of index $k$ by $c_{k}$. One has that $H \Delta(P) \cong C H(M)=H_{*}(M ; \mathbb{Z})$, since $\Delta$ is a connection matrix; and $C_{k} \Delta(P)=\bigoplus C H_{k}\left(M_{x}\right)$, where the sum is over all critical points of $f$. Therefore,

$$
\beta_{k}=\operatorname{rank} H_{k} \Delta(P)=\operatorname{rank} \frac{\operatorname{Ker} \Delta_{k}(P)}{\operatorname{Im} \Delta_{k+1}(P)} \leq \operatorname{rank}\left[\operatorname{Ker} \Delta_{k}(P)\right]=c_{k},
$$

which is precisely the classic Morse inequalities. Furthermore, the equalities hold if and only if $\Delta \equiv 0$. Moreover, if the equality $\beta_{k}=c_{k}$ holds, for all $k=1, \ldots, n=\operatorname{dim}(M)$, then the Morse function $f$ is called a perfect Morse function. Hence, we have proved the following result:

Proposition 2.1. Let $f$ be a perfect Morse function on a closed manifold $M$. Consider a flow $\varphi_{f}$ associated to the vector field $-\nabla f$ and the finest Morse decomposition $\mathcal{D}(M)$ of $M$ with respect to this flow. Then, the set of connection matrices of $\mathcal{D}(M)$ contains only the null map.

$\left.{ }^{1}\right) H_{*}(M ; \mathbb{Z})$ denotes the singular homology with integer coefficients of $M$. 


\section{Connection matrices for Coarser Morse decompositions}

The motivation for this section can be seen in the following hypothetical situation. Given an isolated invariant set $S$ and a Morse decomposition $\mathcal{D}(S)$, one can consider a coarser Morse decomposition $\widetilde{\mathcal{D}}(S)$ of $S$ relative to $\mathcal{D}(S)$. An important question is if there exists any relation between connection matrices of $\mathcal{D}(S)$ and connection matrices of $\widetilde{\mathcal{D}}(S)$. Our goal is to describe the relationship among connection matrices of $\mathcal{D}(S)$ and $\widetilde{\mathcal{D}}(S)$. For instance, let $\mathcal{D}(S)=\left\{M_{1}, \ldots, M_{7}\right\}$ and let $\widetilde{\mathcal{D}}(S)$ be the Morse decomposition obtained when one groups $M_{3}, M_{4}$ and $M_{5}$ as well as their connections in one Morse set $M_{I}$, i.e., $\widetilde{\mathcal{D}}(S)=\left\{M_{1}, M_{2}, M_{I}, M_{6}, M_{7}\right\}$. This grouping will be described in more detail subsequently. Assume that there is an isomorphism $F_{I}$ from $C H\left(M_{I}\right)$ to $C H\left(M_{3}\right) \oplus C H\left(M_{4}\right) \oplus C H\left(M_{5}\right)$ and let

$$
\Delta=\left[\begin{array}{ccccccc}
0 & \Delta(2,1) & \Delta(3,1) & \Delta(4,1) & \Delta(5,1) & \Delta(6,1) & \Delta(7,1) \\
0 & 0 & \Delta(3,2) & \Delta(4,2) & \Delta(5,2) & \Delta(6,2) & \Delta(7,2) \\
0 & 0 & 0 & \Delta(4,3) & \Delta(5,3) & \Delta(6,3) & \Delta(7,3) \\
0 & 0 & 0 & 0 & \Delta(5,4) & \Delta(6,4) & \Delta(7,4) \\
0 & 0 & 0 & 0 & 0 & \Delta(6,5) & \Delta(7,5) \\
0 & 0 & 0 & 0 & 0 & 0 & \Delta(7,6) \\
0 & 0 & 0 & 0 & 0 & 0 & 0
\end{array}\right]
$$

be a connection matrix of $\mathcal{D}(S)$. One can induce a connection matrix $\widetilde{\Delta}$ of the coarser Morse decomposition $\widetilde{\mathcal{D}}(S)$ from $\Delta$, as follows:

$$
\widetilde{\Delta}=\left[\begin{array}{ccccc}
0 & \Delta(2,1) & \widetilde{\Delta}(I, 1) & \Delta(6,1) & \Delta(7,1) \\
0 & 0 & \widetilde{\Delta}(I, 2) & \Delta(6,2) & \Delta(7,2) \\
0 & 0 & 0 & \widetilde{\Delta}(6, I) & \widetilde{\Delta}(7, I) \\
0 & 0 & 0 & 0 & \widetilde{\Delta}(7,6) \\
0 & 0 & 0 & 0 & 0
\end{array}\right]
$$

where the maps $\widetilde{\Delta}(\cdot, \cdot)$ are given by:

$\widetilde{\Delta}(I, j)=\left[\begin{array}{lll}\Delta(3, j) & \Delta(4, j) & \Delta(5, j)\end{array}\right] \circ F_{I} \quad$ and $\quad \widetilde{\Delta}(k, I)=F_{I} \circ\left[\begin{array}{c}\Delta(k, 3) \\ \Delta(k, 4) \\ \Delta(k, 5)\end{array}\right]$

for $j=1,2$ and $k=6,7$.

In what follows we intend to formalize this approach.

Consider a partially ordered set $(P,<)$. Let $I_{1}, \ldots, I_{k}$ be a collection of mutually disjoint intervals with respect to $(P,<)$, such that, if $i<j$ then there are no elements $\pi \in I_{j}$ and $\pi^{\prime} \in I_{i}$ with $\pi<\pi^{\prime}$. In what follows, a subset $\widetilde{P}$ of $\mathcal{I}(P,<)$ is defined to be $\widetilde{P}_{1} \cup \widetilde{P}_{2}$, where $\widetilde{P}_{2}=\left\{\widetilde{\pi} \mid \widetilde{\pi}=I_{j}, j=1, \ldots, k\right\}$ and $\widetilde{P}_{1}=\left\{\widetilde{\pi} \mid \widetilde{\pi}=\{\pi\}\right.$ such that $\left.\pi \in P \backslash\left(I_{1} \cup \ldots \cup I_{k}\right)\right\}$. Hence, the set $\widetilde{P}=\widetilde{P}_{1} \cup \widetilde{P}_{2}$ is composed by intervals of $(P,<)$. It is important to note that $\tilde{\pi}$ is an element 
of $\widetilde{P}$ and is not an element of $P$, but an interval in $(P,<)$. From now on, an element of $\widetilde{P}$ will be denoted by $\widetilde{\pi}$. Although $\widetilde{P}$ is a subset of $\mathcal{I}(<)$ and the elements of $\mathcal{I}(<)$ are usually denoted by $I, J, K$, we will denote elements of $\widetilde{P}$ by $\widetilde{\pi}$ and intervals of $\widetilde{P}$ by $\widetilde{I}, \widetilde{J}, \widetilde{K}$. We also adopt a loose notation $\pi \in \widetilde{\pi}$ to indicate that the element $\pi \in P$ belongs either to the interval $I_{j}$ for $j=1, \ldots, k$ or to the singleton interval in $(P,<)$ composed by itself.

Consider the transitive closure of the relation $\prec$ in $\widetilde{P}$ given by:

$\widetilde{\pi}_{1} \prec \widetilde{\pi}_{2}$, if there are $\pi_{1} \in \widetilde{\pi}_{1}$ and $\pi_{2} \in \widetilde{\pi}_{2}$ such that $\pi_{1}<\pi_{2}$

where $\widetilde{\pi}_{1}, \widetilde{\pi}_{2} \in \widetilde{P}$ and $\widetilde{\pi}_{1} \neq \widetilde{\pi}_{2}$.

Proposition 3.1. The pair $(\widetilde{P}, \prec)$ is a partially ordered set.

Proof. One needs to prove that $\widetilde{\pi} \nprec \widetilde{\pi}$ for all $\widetilde{\pi} \in \widetilde{P}$. Suppose by contradiction that $\widetilde{\pi} \prec \widetilde{\pi}$. Hence, by definition of the relation $\prec$, there exists $\widetilde{\pi}^{\prime} \in \widetilde{P}$ such that $\widetilde{\pi} \prec \widetilde{\pi}^{\prime} \prec \widetilde{\pi}$. If $\widetilde{\pi}^{\prime}$ is a singleton, i.e. $\widetilde{\pi}^{\prime}=\left\{\pi^{\prime}\right\} \in \widetilde{P}_{1}$, then there must exist $\pi_{1}, \pi_{2} \in \tilde{\pi}$ such that $\pi_{1}<\pi^{\prime}<\pi_{2}$. But $\widetilde{\pi} \in \mathcal{I}(P,<)$, then $\pi^{\prime} \in \tilde{\pi}$, which contradicts the fact that $\widetilde{\pi}^{\prime} \in \widetilde{P}_{1}$. Therefore, $\widetilde{\pi}^{\prime}$ is not a singleton, that is, $\widetilde{\pi}^{\prime}=I_{j} \in \widetilde{P}_{2}$, for some $j=1, \ldots, k$. Hence, one has that $\widetilde{\pi} \prec \widetilde{\pi}^{\prime}=I_{j} \prec \widetilde{\pi}$, which contradicts the choices of the intervals $I_{1}, \ldots, I_{k}$.

The next proposition relates intervals in $(\widetilde{P}, \prec)$ with intervals in $(P,<)$. Given an interval $\widetilde{J}$ in $(\widetilde{P}, \prec)$, define $J$ to be the subset of $P$ such that $\pi \in J$ iff $\pi \in \widetilde{\pi}$ for some $\widetilde{\pi} \in \widetilde{J}$. The set $J$ is well defined since each element $\widetilde{\pi}$ of $\widetilde{J}$ is an interval of $(P,<)$.

Proposition 3.2.

(a) If $\widetilde{J} \in \mathcal{I}(\widetilde{P}, \prec)$ then $J \in \mathcal{I}(P,<)$.

(b) If $(\widetilde{J}, \widetilde{K}) \in \mathcal{I}_{2}(\widetilde{P}, \prec)$ then $(J, K) \in \mathcal{I}_{2}(P,<)$.

Proof. (a) Let $\widetilde{J}$ be an interval in $(\widetilde{P}, \prec)$. Given $\pi_{1}, \pi_{2} \in J$ and $\pi \in P$ such that $\pi_{1}<\pi<\pi_{2}$, one must show that $\pi \in J$. In fact, there must exist $\widetilde{\pi}_{1}, \widetilde{\pi}_{2}, \widetilde{\pi} \in \widetilde{P}$ such that $\pi_{1} \in \widetilde{\pi}_{1}, \pi_{2} \in \widetilde{\pi}_{2}$ and $\pi \in \widetilde{\pi}$. Hence, $\widetilde{\pi}_{1} \prec \widetilde{\pi} \prec \widetilde{\pi}_{2}$, which implies that $\widetilde{\pi} \in \widetilde{J}$. Therefore, $\pi \in J$.

(b) As $\widetilde{J} \cup \widetilde{K}$ is a interval in $(\widetilde{P}, \prec)$, by the previous item, $J \cup K$ is an interval in $(P,<)$. Suppose by contradiction that $\pi_{1} \in J, \pi_{2} \in K$ and $\pi_{2}<\pi_{1}$. By definition of $J, K$, there exist $\widetilde{\pi}_{1} \in \widetilde{J}$ and $\widetilde{\pi}_{2} \in \widetilde{K}$ such that $\pi_{1} \in \widetilde{\pi}_{1}$ and $\pi_{2} \in \widetilde{\pi}_{2}$. Hence, $\widetilde{\pi}_{2} \prec \widetilde{\pi}_{1}$, which contradicts the fact that $(\widetilde{J}, \widetilde{K})$ is an adjacent pair of intervals.

Indeed, one can show that if $\left(\widetilde{J}_{1}, \ldots, \widetilde{J}_{n}\right) \in \mathcal{I}_{n}(\widetilde{I}, \prec)$ then $\left(J_{1}, \ldots, J_{n}\right) \in$ $\mathcal{I}_{n}(I, \prec)$. The proof follows the same ideas of the proof of Proposition 3.2(b).

Let $S$ be an isolated invariant set and $\mathcal{D}(S)=\left\{M_{\pi}: \pi \in P\right\}$ be a Morse decomposition of $S$ with admissible ordering $<$. The purpose of this section is to 
study connection matrices with coarser Morse decompositions of $S, \widetilde{\mathcal{D}}(S)$. This is done by considering as Morse sets of $\widetilde{\mathcal{D}}(S)$ the union of some $M_{\pi}$ of $\mathcal{D}(S)$ and their connections. More specifically, let $\widetilde{\mathcal{D}}(S)=\left\{\widetilde{M}_{\widetilde{\pi}}: \widetilde{\pi} \in \widetilde{P}\right\}$, where

$$
\widetilde{M}_{\widetilde{\pi}}=M_{\widetilde{\pi}}=\left(\bigcup_{\pi \in \widetilde{\pi}} M_{\pi}\right) \cup\left(\bigcup_{\pi, \pi^{\prime} \in \widetilde{\pi}} C\left(M_{\pi^{\prime}}, M_{\pi}\right)\right) \text {. }
$$

$\widetilde{\mathcal{D}}(S)$ is not just a collection of some Morse sets of $P$ with respect to $<$, it is an $\prec$-ordered Morse decomposition of $S$. See Proposition 3.3 below. Before proving this, we present a characterization of the set of orbits connecting two Morse sets in the coarser Morse decomposition $\widetilde{\mathcal{D}}(S)$ by means of the set of orbits connecting two isolated invariant set in the original Morse decomposition $\mathcal{D}(S)$. Let $\widetilde{\pi}_{1}, \widetilde{\pi}_{2} \in \widetilde{P}$, the set of orbits connecting $\widetilde{M}_{\widetilde{\pi}_{2}}$ to $\widetilde{M}_{\widetilde{\pi}_{1}}, C\left(\widetilde{M}_{\widetilde{\pi}_{2}}, \widetilde{M}_{\widetilde{\pi}_{1}}\right)$, is given by

$$
C\left(\widetilde{M}_{\widetilde{\pi}_{2}}, \widetilde{M}_{\widetilde{\pi}_{1}}\right)=\bigcup_{\pi^{\prime} \in \widetilde{\pi}_{2}, \pi \in \widetilde{\pi}_{1}} C\left(M_{\pi^{\prime}}, M_{\pi}\right) .
$$

This characterization is essentially the proof of the following two propositions.

Proposition 3.3. The set $\widetilde{\mathcal{D}}(S)$ is a Morse decomposition of $S$ with admissible ordering $\prec$.

Proof. The sets $\widetilde{M}_{\widetilde{\pi}}$ are isolated, invariant, disjoint and compact, by definition. If $\gamma \in S$ and $\gamma \notin \underset{\widetilde{\pi} \in \widetilde{P}}{\bigcup} \widetilde{M}_{\widetilde{\pi}}$ one must prove that $\gamma \in C\left(\widetilde{M}_{\widetilde{\pi}}, \widetilde{M}_{\widetilde{\pi}^{\prime}}\right)$ with $\tilde{\pi}^{\prime} \prec \widetilde{\pi}$. Observe that $\gamma \notin \bigcup_{\pi \in P}^{\pi \in \widetilde{P}} M_{\pi}$ and, as $\mathcal{D}(S)$ is a Morse decomposition of $S$, there exist $\pi_{1}, \pi_{2} \in P$ such that $\pi_{1}<\pi_{2}$ and $\gamma \in C\left(M_{\pi_{2}}, M_{\pi_{1}}\right)$. Therefore, $\gamma \in C\left(\widetilde{M}_{\widetilde{\pi}}, \widetilde{M}_{\widetilde{\pi}^{\prime}}\right)$, for $\widetilde{\pi}^{\prime}, \widetilde{\pi} \in \widetilde{P}$ such that $\pi_{1} \in \widetilde{\pi}$ and $\pi_{2} \in \widetilde{\pi}^{\prime}$.

Proposition 3.4. If < induces the flow ordering of the Morse decomposition $\mathcal{D}(S)$, then $\prec$ induces the flow ordering of the coarser Morse decomposition $\widetilde{\mathcal{D}}(S)$.

Proof. Given $\widetilde{\pi}, \widetilde{\pi}^{\prime} \in \widetilde{P}$ with $\widetilde{\pi} \prec \widetilde{\pi}^{\prime}$, one needs to show that there exist $\widetilde{\pi}=\widetilde{\pi}_{0}, \widetilde{\pi}_{1}, \ldots, \widetilde{\pi}_{n-1}, \widetilde{\pi}_{n}=\widetilde{\pi}^{\prime}$ in $\widetilde{P}$ such that $C\left(\widetilde{M}_{\widetilde{\pi}_{i}}, \widetilde{M}_{\widetilde{\pi}_{i-1}}\right) \neq \emptyset$. This proof is straightforward and is done by analysing the possibilities of $\pi, \pi^{\prime} \in \widetilde{P}$ as elements of $\widetilde{P}_{1}$ or $\widetilde{P}_{2}$ and using the characterization of the set of orbits connecting $\widetilde{M}_{\pi^{\prime}}$ to $\widetilde{M}_{\pi}$.

Having defined the Morse decomposition $\widetilde{D}(S)$ with admissible ordering $\prec$, one can now define connection matrices in this setting.

Let $\mathcal{H}(<)=\mathcal{H}(<; G)$ be the homology index braid of $<$ with coefficients in $G$. Let $C=\{C \Delta(\pi)\}_{\pi \in P}$ be a collection of free chain complexes with trivial boundary operator, where $C \Delta(\pi)=C H\left(M_{\pi}\right)$ is the Conley homological index of $M_{\pi}$ with coefficients in $G$, for all $\pi \in P$. Therefore, the collection of connection matrices of $\mathcal{H}(<), \mathcal{C M}(\mathcal{H}(<))$, is non empty, since the graded module braid $\mathcal{H}(<)$ is a chain complex generated. 
From a given connection matrix $\Delta: C \Delta(P) \rightarrow C \Delta(P)$ of $\mathcal{H}(<)$, we will construct a connection matrix $\widetilde{\Delta}: C \widetilde{\Delta}(\widetilde{P}) \rightarrow C \widetilde{\Delta}(\widetilde{P})$ of the homological index braid of $\prec$ with coefficients in $G, \mathcal{H}(\prec)=\mathcal{H}(\prec ; G)$. In order to do this, we will henceforth assume that, for each interval $I_{1}, \ldots, I_{k}$ previously set in $(P,<)$, $\Delta\left(I_{j}\right)=0$. Note that, for other intervals in $(P,<)$ different from $I_{1}, \ldots, I_{k}$, this assumption is not required. Using this assumption, it follows that

$$
H \Delta\left(I_{j}\right)=\frac{\operatorname{Ker} \Delta\left(I_{j}\right)}{\operatorname{Im} \Delta\left(I_{j}\right)}=\frac{\bigoplus_{\pi \in I_{j}} C H\left(M_{\pi}\right)}{0} \cong \bigoplus_{\pi \in I_{j}} C H\left(M_{\pi}\right)
$$

for all $j=1, \ldots, k$. As $\Delta$ is a connection matrix of $\mathcal{H}(<)$ then $C H\left(M_{I_{j}}\right) \cong$ $H \Delta\left(I_{j}\right)$, for all $j=1, \ldots, k$, and one has

$$
C H\left(M_{I_{j}}\right) \cong \bigoplus_{\pi \in I_{j}} C H\left(M_{\pi}\right) .
$$

Now, let $\widetilde{C}=\{C \widetilde{\Delta}(\widetilde{\pi})\}_{\widetilde{\pi} \in \widetilde{P}}$ be a chain complex braid over $\prec$, where $C \widetilde{\Delta}(\widetilde{\pi})=$ $C H\left(\widetilde{M}_{\widetilde{\pi}}\right)$, for each $\widetilde{\pi} \in \widetilde{P}$, and the boundary operator is trivial. The chain complex braids $C=\{C \Delta(\pi)\}_{\pi \in P}$ and $\widetilde{C}=\{C \widetilde{\Delta}(\widetilde{\pi})\}_{\widetilde{\pi} \in \widetilde{P}}$ are related to each other by:

$$
\begin{gathered}
C \widetilde{\Delta}(\{\pi\})=C H\left(\widetilde{M}_{\{\pi\}}\right)=C H\left(M_{\pi}\right)=C \Delta(\pi) ; \\
C \widetilde{\Delta}\left(I_{j}\right)=C H\left(\widetilde{M}_{I_{j}}\right)=C H\left(M_{I_{j}}\right) \cong \bigoplus_{\pi^{\prime} \in I_{j}} C H\left(M_{\pi^{\prime}}\right)=\bigoplus_{\pi^{\prime} \in I_{j}} C \Delta\left(\pi^{\prime}\right)=C \Delta\left(I_{j}\right) .
\end{gathered}
$$

In short, $C \widetilde{\Delta}(\widetilde{\pi})=C \Delta(\widetilde{\pi})$.

For each $\tilde{\pi} \in \widetilde{P}$, let

$$
F_{\widetilde{\pi}}: C \widetilde{\Delta}(\widetilde{\pi}) \rightarrow C \Delta(\widetilde{\pi})
$$

be an isomorphism. If $\widetilde{\pi} \in \widetilde{P}_{1}$, i.e. $\widetilde{\pi}$ is a singleton, we consider $F_{\widetilde{\pi}}$ as be the identity map.

The set of connection matrices of $\mathcal{H}(\prec, G)$ is non empty, since this graded module braid is chain complex generated. Now, from $\Delta$ we will make explicit a connection matrix of $\mathcal{H}(\prec, G)$. Let $\widetilde{\Delta}: C \widetilde{\Delta}(\widetilde{P}) \rightarrow C \widetilde{\Delta}(\widetilde{P})$ be the map regarded as a matrix

$$
\widetilde{\Delta}=\left(\widetilde{\Delta}\left(\widetilde{\pi}^{\prime}, \widetilde{\pi}\right)\right)_{\tilde{\pi}^{\prime}, \tilde{\pi}^{\prime} \in \widetilde{P}},
$$

where each $\widetilde{\Delta}\left(\widetilde{\pi}^{\prime}, \widetilde{\pi}\right)$ is the map from $C \widetilde{\Delta}\left(\widetilde{\pi}^{\prime}\right)$ to $C \widetilde{\Delta}(\widetilde{\pi})$ defined as follows:

$$
\widetilde{\Delta}_{\widetilde{\pi}^{\prime}, \tilde{\pi}}=F_{\widetilde{\pi}}^{-1} \circ \Delta\left(\widetilde{\pi}^{\prime}, \widetilde{\pi}\right) \circ F_{\widetilde{\pi}^{\prime}} .
$$

The goal in this section is to show that $\widetilde{\Delta}=\widetilde{\Delta}(\widetilde{P})$ is a connection matrix of $\mathcal{H}(\prec)$, which will be proved in Theorem 3.7. The next result describes a relation between the maps $\widetilde{\Delta}(\widetilde{K}, \widetilde{J})$ and $\Delta(K, J)$, for each $(\widetilde{J}, \widetilde{K})$ adjacent pair of intervals in $(\widetilde{P}, \prec)$. This result is essential to the proof of the main theorem. 
TheOREM 3.5. Given a pair of adjacent intervals $(\widetilde{J}, \widetilde{K}) \in \mathcal{I}_{2}(\widetilde{P}, \prec)$, the map $\widetilde{\Delta}(\widetilde{K}, \widetilde{J})$ is conjugated to $\Delta(K, J)$, that is, there exist isomorphisms $R_{J}: C \widetilde{\Delta}(\widetilde{J})$ $\rightarrow C \Delta(J)$ and $R_{K}: C \widetilde{\Delta}(\widetilde{K}) \rightarrow C \Delta(K)$ such that

$$
\widetilde{\Delta}(\widetilde{K}, \widetilde{J})=R_{J}^{-1} \circ \Delta(K, J) \circ R_{K} .
$$

In particular, for each $\widetilde{J} \in \mathcal{I}(\widetilde{P}, \prec)$,

$$
\widetilde{\Delta}(\widetilde{J})=R_{J}^{-1} \circ \Delta(J) \circ R_{J} .
$$

Proof. Let $\widetilde{J} \in \mathcal{I}(\widetilde{P}, \prec)$ and $\left\{I_{j_{1}}, \ldots, I_{j_{p}}\right\}$ the elements in the intersection $\widetilde{J} \cap \widetilde{P}_{2}$. To simplify notation, renumber these elements as $I_{1}, \ldots, I_{p}$, such that, if $I_{i} \prec I_{j}$ then $i<j$. Define the following subsets of $\widetilde{J}$ :

$$
\begin{aligned}
B_{\ell}= & \left\{\widetilde{\pi} \in \widetilde{J}: \widetilde{\pi} \prec I_{\ell+1}\right\} \backslash\left(B_{0} \cup \ldots \cup B_{\ell}\right), \text { for } \ell=0, \ldots, p-1, \\
B_{p}= & \left\{\widetilde{\pi} \in \widetilde{J}: I_{p} \prec \widetilde{\pi}\right\} \\
& \cup\left\{\widetilde{\pi} \in \widetilde{J}: \widetilde{\pi} \text { and } I_{i} \text { are noncomparable for all } i=1, \ldots, p\right\} .
\end{aligned}
$$

Note that $B_{i} \cap B_{j}=\emptyset$ if $i \neq j$ and $\left(\bigcup_{i=0}^{p} B_{i}\right) \cup\left(\bigcup_{i=1}^{p} I_{i}\right)=\widetilde{J}$. This collection of subsets of $\widetilde{J}$ has the following properties:

(a) $B_{i} \in \mathcal{I}(\widetilde{P}, \prec)$, for $i=0, \ldots, p$;

(b) $\left(B_{0}, I_{1}\right),\left(B_{k}, I_{k+1}\right),\left(I_{k}, B_{k}\right) \in \mathcal{I}_{2}(\widetilde{P}, \prec)$, for all $k=1, \ldots, p$;

(c) $\left(B_{0}, I_{1}, B_{1}, \ldots, I_{p}, B_{p}\right) \in \mathcal{I}_{2 p+1}(\widetilde{P}, \prec)$.

Given $(\widetilde{J}, \widetilde{K}) \in \mathcal{I}_{2}(\widetilde{P}, \prec)$, let $I_{i_{1}}^{J}, \ldots, I_{i_{p}}^{J} \in \widetilde{J}$ and $I_{i_{1}}^{K}, \ldots, I_{i_{q}}^{K} \in \widetilde{K}\left({ }^{2}\right)$ be the only elements in $\widetilde{J} \cap \widetilde{P}_{2}$ and $\widetilde{K} \cap \widetilde{P}_{2}$, respectively. Renumber these elements as $I_{1}^{J}, \ldots, I_{p}^{J}$ and $I_{1}^{K}, \ldots, I_{q}^{K}$ such that, if $I_{i}^{J} \prec I_{j}^{J}$ then $i<j$, and if $I_{i}^{K} \prec$ $I_{j}^{K}$ then $i<j$. Consider the decompositions $\left(B_{0}, I_{1}^{J}, B_{1}, \ldots, I_{p}^{J}, B_{p}\right)$ of $\widetilde{J}$ and $\left(A_{0}, I_{1}^{K}, A_{1}, \ldots, I_{q}^{K}, A_{q}\right)$ of $\widetilde{K}$, as described above. Using these decompositions, one can view $\widetilde{\Delta}$ as a map given by the matrix

$$
\begin{aligned}
& \widetilde{\Delta}(\widetilde{K}, \widetilde{J}) \\
& =\left[\begin{array}{cccccc}
\widetilde{\Delta}\left(A_{0}, B_{0}\right) & \widetilde{\Delta}\left(I_{1}^{K}, B_{0}\right) & \widetilde{\Delta}\left(A_{1}, B_{0}\right) & \cdots & \widetilde{\Delta}\left(I_{q}^{K}, B_{0}\right) & \widetilde{\Delta}\left(A_{q}, B_{0}\right) \\
\widetilde{\Delta}\left(A_{0}, I_{1}^{J}\right) & \widetilde{\Delta}\left(I_{1}^{K}, I_{1}^{J}\right) & \widetilde{\Delta}\left(A_{1}, I_{1}^{J}\right) & \cdots & \widetilde{\Delta}\left(I_{q}^{K}, I_{1}^{J}\right) & \widetilde{\Delta}\left(A_{q}, I_{1}^{J}\right) \\
\widetilde{\Delta}\left(A_{0}, B_{1}\right) & \widetilde{\Delta}\left(I_{1}^{K}, B_{1}\right) & \widetilde{\Delta}\left(A_{1}, B_{1}\right) & \cdots & \widetilde{\Delta}\left(I_{q}^{K}, B_{1}\right) & \widetilde{\Delta}\left(A_{q}, B_{1}\right) \\
\vdots & \vdots & \vdots & \ddots & \vdots & \vdots \\
\widetilde{\Delta}\left(A_{0}, I_{p}^{J}\right) & \widetilde{\Delta}\left(I_{1}^{K}, I_{p}^{J}\right) & \widetilde{\Delta}\left(A_{1}, I_{p}^{J}\right) & \cdots & \widetilde{\Delta}\left(I_{q}^{K}, I_{p}^{J}\right) & \widetilde{\Delta}\left(A_{q}, I_{p}^{J}\right) \\
\widetilde{\Delta}\left(A_{0}, B_{p}\right) & \widetilde{\Delta}\left(I_{1}^{K}, B_{p}\right) & \widetilde{\Delta}\left(A_{1}, B_{p}\right) & \cdots & \widetilde{\Delta}\left(I_{q}^{K}, B_{p}\right) & \widetilde{\Delta}\left(A_{q}, B_{p}\right)
\end{array}\right] .
\end{aligned}
$$

$\left({ }^{2}\right)$ The superscript $J$ (resp. $K$ ) is in order to clarify the relation of elements belonging to $\widetilde{J}(\operatorname{resp} . \widetilde{K})$. 
For each $\widetilde{J} \in \mathcal{I}(\widetilde{P}, \prec)$, consider the isomorphism $R_{J}$ from

$$
C \widetilde{\Delta}(\widetilde{J})=\bigoplus_{\pi \in B_{0}} C \widetilde{\Delta}(\pi) \oplus C \widetilde{\Delta}\left(I_{1}\right) \oplus \ldots \oplus \bigoplus_{\pi \in B_{p-1}} C \widetilde{\Delta}(\pi) \oplus C \widetilde{\Delta}\left(I_{p}\right) \bigoplus_{\pi \in B_{p}} C \widetilde{\Delta}(\pi)
$$

to

$$
C \Delta(J)=\bigoplus_{\pi \in B_{0}} C \Delta(\pi) \bigoplus_{\pi \in I_{1}} C \Delta(\pi) \oplus \ldots \oplus \bigoplus_{\pi \in B_{p-1}} C \Delta(\pi) \bigoplus_{\pi \in I_{p}} C \Delta(\pi) \bigoplus_{\pi \in B_{p}} C \Delta(\pi),
$$

given by

$$
R_{J}=\left(\mathrm{id}, F_{I_{1}}, \mathrm{id}, F_{I_{2}}, \ldots, \mathrm{id}, F_{I_{p}}, \mathrm{id}\right),
$$

where $F_{I_{j}}$ is the isomorphisms defined in (3.1). Using this matrix notation, it is not difficult to see that $\widetilde{\Delta}(\widetilde{K}, \widetilde{J})=R_{J}^{-1} \circ \Delta(K, J) \circ R_{K}$.

If $\widetilde{J}$ (resp. $\widetilde{K})$ contains no elements of $\widetilde{P}_{2}$, it is also true that $\widetilde{\Delta}(\widetilde{K}, \widetilde{J})=$ $R_{J}^{-1} \circ \Delta(K, J) \circ R_{K}$, where $R_{J}=\mathrm{id}\left(R_{K}=\mathrm{id}\right.$, respectively).

To see that $\widetilde{\Delta}(\widetilde{J})=R_{J}^{-1} \circ \Delta(J) \circ R_{J}$, for a given $\widetilde{J} \in \mathcal{I}(\widetilde{P}, \prec)$, just consider the decomposition $\left(B_{0}, I_{1}, B_{1}, \ldots, I_{p}, B_{p}\right)$ of $\widetilde{J}$, as described above, and visualize the map $\widetilde{\Delta}(\widetilde{J})$ as

$$
\widetilde{\Delta}(\widetilde{J})=\left[\begin{array}{cccccc}
\widetilde{\Delta}\left(B_{0}\right) & \widetilde{\Delta}\left(I_{1}, B_{0}\right) & \widetilde{\Delta}\left(B_{1}, B_{0}\right) & \ldots & \widetilde{\Delta}\left(I_{p}, B_{0}\right) & \widetilde{\Delta}\left(B_{p}, B_{0}\right) \\
0 & \widetilde{\Delta}\left(I_{1}\right) & \widetilde{\Delta}\left(B_{1}, I_{1}\right) & \cdots & \widetilde{\Delta}\left(I_{p}, I_{1}\right) & \widetilde{\Delta}\left(B_{p}, I_{1}\right) \\
0 & 0 & \widetilde{\Delta}\left(B_{1}\right) & \ldots & \widetilde{\Delta}\left(I_{p}, B_{1}\right) & \widetilde{\Delta}\left(B_{p}, B_{1}\right) \\
\vdots & \vdots & \vdots & \ddots & \vdots & \vdots \\
0 & 0 & 0 & \cdots & \widetilde{\Delta}\left(I_{p}\right) & \widetilde{\Delta}\left(B_{p}, I_{p}\right) \\
0 & 0 & 0 & \cdots & 0 & \widetilde{\Delta}\left(B_{p}\right)
\end{array}\right] .
$$

If $\widetilde{J}$ contains none of the elements $I_{1}, \ldots, I_{k} \in \widetilde{P}_{2}$, then $\widetilde{\Delta}(\widetilde{J})=\Delta(J)$, by definition of the map $\widetilde{\Delta}$.

The first step to show that $\widetilde{\Delta}$ is a connection matrix of $\mathcal{H}(\prec)$ is to check if $\widetilde{\Delta}$ is a strictly upper triangular boundary map, since all connection matrices have this property.

THEOREM 3.6.

(a) The map $\widetilde{\Delta}$ is strictly upper triangular, i.e. $\widetilde{\Delta}\left(\widetilde{\pi}^{\prime}, \widetilde{\pi}\right) \neq 0$ implies $\widetilde{\pi} \prec \widetilde{\pi}^{\prime}$;

(b) The map $\widetilde{\Delta}$ is a boundary map, i.e. $\widetilde{\Delta}\left(\widetilde{\pi}^{\prime}, \widetilde{\pi}\right)$ is of degree -1 and $\widetilde{\Delta} \circ \widetilde{\Delta}=0$.

Proof. (a) Suppose that $\widetilde{\Delta}\left(\widetilde{\pi}^{\prime}, \widetilde{\pi}\right) \neq 0$. By definition of $\widetilde{\Delta}$ in (3.2), one has that $\Delta\left(\widetilde{\pi}^{\prime}, \tilde{\pi}\right) \neq 0$. Hence, there are $\pi_{1} \in \tilde{\pi}, \pi_{2} \in \widetilde{\pi}^{\prime}$ such that $\Delta\left(\pi_{2}, \pi_{1}\right) \neq 0$. Therefore, $\pi_{1}<\pi_{2}$, which implies $\widetilde{\pi} \prec \widetilde{\pi}^{\prime}$.

(b) By definition of $\widetilde{\Delta}$ in (3.2), it follows that $\widetilde{\Delta}\left(\widetilde{\pi}^{\prime}, \widetilde{\pi}\right)$ is of degree -1 , for all $\widetilde{\pi}, \widetilde{\pi}^{\prime} \in \widetilde{P}$. By Theorem 3.5, one has that for each interval $\widetilde{J} \in(\widetilde{P}, \prec)$ :

$$
\widetilde{\Delta}(\widetilde{J})^{2}=\left(R_{J}^{-1} \circ \Delta(J) \circ R_{J}\right)\left(R_{J}^{-1} \circ \Delta(J) \circ R_{J}\right)=\left(R_{J}^{-1} \circ \Delta(J)^{2} \circ R_{J}\right),
$$

which is zero, since $\Delta(J)$ is a boundary map. In particular, $\widetilde{\Delta}(\widetilde{P})^{2}=0$. 
We are now able to prove our main theorem.

TheOrem 3.7. The map $\widetilde{\Delta}: C \widetilde{P} \rightarrow C \widetilde{P}$ is a connection matrix of $\mathcal{H}(\prec)$.

Proof. By Theorem 3.6, $\widetilde{\Delta}$ is a strictly upper triangular boundary map. To show that this map is a connection matrix of $\mathcal{H}(\prec)$, one needs to guarantee that the graded module braid $\mathcal{H} \widetilde{\Delta}$ is isomorphic to the homology index braid $\mathcal{H}(\prec)$.

As $\Delta$ is a connection matrix, then the graded module braid $\mathcal{H} \Delta$ is isomorphic to the homology index braid $\mathcal{H}(<)$. Moreover, for each $\widetilde{J} \in \mathcal{I}_{2}(\widetilde{P}, \prec)$, one has that $C H\left(\widetilde{M}_{\widetilde{J}}\right) \cong C H\left(M_{J}\right)$. Hence, to prove this theorem, it is sufficient to show that $\mathcal{H} \widetilde{\Delta}$ is isomorphic to $\mathcal{H} \Delta$, i.e. that there exists a collection of isomorphisms $\Psi(\widetilde{J}): H \widetilde{\Delta}(\widetilde{J}) \rightarrow H \Delta(J), \widetilde{J} \in \mathcal{I}(\widetilde{P}, \prec)$, such that, for each $(\widetilde{J}, \widetilde{K}) \in \mathcal{I}_{2}(\widetilde{P}, \prec)$ the following diagram commutes:

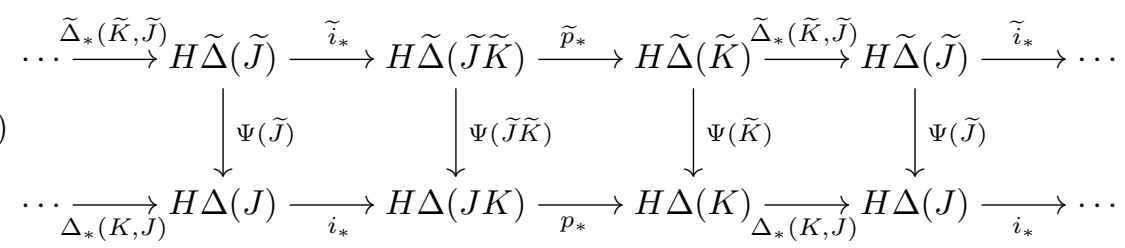

We will start by showing that, for each $\widetilde{J} \in \mathcal{I}(\widetilde{P}, \prec), H \widetilde{\Delta}(\widetilde{J})$ and $H \Delta(J)$ are isomorphic by constructing an isomorphism $\Psi(\widetilde{J})$. Afterwards, we will use this collection of isomorphisms to prove that the diagram in (3.4) is commutative.

For each $\widetilde{J} \in \mathcal{I}(\widetilde{P}, \prec)$, let $\Psi(\widetilde{J}): H \widetilde{\Delta}(\widetilde{J}) \rightarrow H \Delta(J)$ be the map induced from the collection $\left\{F_{\pi}\right\}_{\pi \in \widetilde{P}}$ on the quotient modules $H \widetilde{\Delta}(\widetilde{J})$ and $H \Delta(J)$. More specifically, considering a decomposition of $\widetilde{J}$ as $\left(B_{0}, I_{1}, B_{1}, \ldots, I_{p}, B_{p}\right)$ (as in the proof of Theorem 3.6), define

$$
\begin{aligned}
\Psi(\widetilde{J}): H \widetilde{\Delta}(\widetilde{J})=\frac{\operatorname{Ker} \widetilde{\Delta}(\widetilde{J})}{\operatorname{Im} \widetilde{\Delta}(\widetilde{J})} & \rightarrow H \Delta(J)=\frac{\operatorname{Ker} \Delta(J)}{\operatorname{Im} \Delta(J)}, \\
{[a] } & \mapsto\left[R_{J}(a)\right],
\end{aligned}
$$

where, $R_{J}: C \widetilde{\Delta}(\widetilde{J}) \rightarrow C \Delta(J)$ is the isomorphism defined in (3.3). Observe that, if $\widetilde{J}$ does not contain elements of $\widetilde{P}_{2}$, then $\Psi(\widetilde{J})$ is the identity map. The following two claims show that $\Psi(\widetilde{J})$ is well defined and is an isomorphism of modules.

Claim 3.8. The map $\Psi(\widetilde{J})$ is well defined.

We need to show that if $[a] \in H \widetilde{\Delta}(\widetilde{J})$ then $\left[R_{J}(a)\right] \in H \Delta(J)$, and that $\Psi(\widetilde{J})$ does not depend on the particular choice of representatives. Firstly, one has that

$$
\begin{aligned}
{[a] \in H \widetilde{\Delta}(\widetilde{J}) } & \Rightarrow a \in \operatorname{Ker} \widetilde{\Delta}(\widetilde{J}) \\
& \Rightarrow R_{J}(a) \in \operatorname{Ker} \Delta(J), \quad \text { since } \widetilde{\Delta}(\widetilde{J})=R_{J}^{-1} \circ \Delta(J) \circ R_{J} \\
& \Rightarrow\left[R_{J}(a)\right] \in H \Delta(J) .
\end{aligned}
$$


This proves that if $[a] \in H \widetilde{\Delta}(\widetilde{J})$ then $\left[R_{J}(a)\right] \in H \Delta(J)$. On the other hand,

$$
\begin{aligned}
{[a]=[b] \in H \widetilde{\Delta}(\widetilde{J}) } & \Rightarrow[a-b]=0 \\
& \Rightarrow a-b \in \operatorname{Im} \widetilde{\Delta}(\widetilde{J}) \\
& \Rightarrow R_{J}(a-b) \in \operatorname{Im} \Delta(J) \quad \text { since } \widetilde{\Delta}(\widetilde{J})=R_{J}^{-1} \circ \Delta(J) \circ R_{J} \\
& \Rightarrow R_{J}(a)-R_{J}(b) \in \operatorname{Im} \Delta(J) \\
& \Rightarrow\left[R_{J}(a)\right]=\left[R_{J}(b)\right] .
\end{aligned}
$$

Hence, $\Psi(\widetilde{J})$ does not depend on the particular choice of representatives.

Claim 3.9. The map $\Psi(\widetilde{J})$ is an isomorphism of modules.

It is not difficult to see that $\Psi(\widetilde{J})$ is a homomorphism of modules. We will prove that it is bijective. Observe that $\Psi(\widetilde{J})$ is injective, since

$$
\begin{aligned}
\Psi(\widetilde{J})[a]= & \Psi(\widetilde{J})[b] \in H \Delta(J) \\
& \Rightarrow\left[R_{J}(a-b)\right]=0, \quad \text { i.e. } R_{J}(a-b) \in \operatorname{Im} \Delta(J) \\
& \Rightarrow a-b \in \operatorname{Im} R_{J}^{-1} \circ \Delta(J) \\
& \Rightarrow a-b \in \operatorname{Im} \widetilde{\Delta}(\widetilde{J}), \quad \text { since } \widetilde{\Delta}(\widetilde{J}) \circ R_{J}^{-1}=R_{J}^{-1} \circ \Delta(J) \\
& \Rightarrow[a]=[b] .
\end{aligned}
$$

Now, given $[b] \in H \Delta(J)$, let $a=R_{J}^{-1}(b)$. Observe that

$$
\widetilde{\Delta}(\widetilde{J})(a)=\widetilde{\Delta}(\widetilde{J}) \circ R_{J}^{-1}(b)=R_{J}^{-1} \circ \Delta(J)(b)=0,
$$

which implies $[a] \in H \widetilde{\Delta}(\widetilde{J})$. Moreover, $\Psi(\widetilde{J})[a]=\Psi(\widetilde{J})\left[R_{J}^{-1}(b)\right]=[b]$, proving that $\Psi(\widetilde{J})$ is surjective.

Therefore, $\Psi(\widetilde{J})$ is an isomorphism between $H \widetilde{\Delta}(\widetilde{J})$ and $H \Delta(J)$, for all intervals $\widetilde{J}$ in $(\widetilde{P}, \prec)$.

Now, considering the family $\{\Psi(\widetilde{J}): \widetilde{J} \in \mathcal{I}(\widetilde{P}, \prec)\}$, we will show that the diagram in (3.4) is commutative, for all pair of adjacent intervals $(\widetilde{J}, \widetilde{K}) \in(\widetilde{P}, \prec)$. Indeed:

$$
\text { - } \Psi(\widetilde{J}) \circ \widetilde{\Delta}_{*}(\widetilde{K}, \widetilde{J})=\Delta_{*}(K, J) \circ \Psi(\widetilde{K}) .
$$

By Theorem 3.5, $\widetilde{\Delta}(\widetilde{K}, \widetilde{J})=R_{J}^{-1} \circ \Delta(K, J) \circ R_{K}$. Therefore, given $[a] \in H \widetilde{\Delta}(\widetilde{K})$, one has

$$
\begin{aligned}
\Psi(\widetilde{J}) \circ \widetilde{\Delta}_{*}(\widetilde{K}, \widetilde{J})[a] & =\Psi(\widetilde{J})[\widetilde{\Delta}(\widetilde{K}, \widetilde{J}) a]=\left[R_{J} \circ \widetilde{\Delta}(\widetilde{K}, \widetilde{J}) a\right] \\
& =\left[\Delta(K, J) \circ R_{K} a\right]=\Delta_{*}(K, J)\left[R_{K} a\right] \\
& =\Delta_{*}(K, J) \circ \Psi(\widetilde{K})[a] .
\end{aligned}
$$

- $\Psi(\widetilde{J} \widetilde{K}) \circ \widetilde{i}_{*}=i_{*} \circ \Psi(\widetilde{J})$. 
The isomorphism $R_{J K}$ restricted to the first component of $C \widetilde{\Delta}(\widetilde{J} \widetilde{K})=C \widetilde{\Delta}(\widetilde{J}) \oplus$ $C \widetilde{\Delta}(\widetilde{K})$ behaves as the isomorphism $R_{J}$, i.e. $\left.R_{J K}\right|_{C \widetilde{\Delta}(\widetilde{J})}=R_{J}$. Thus, $R_{J K} \circ \widetilde{i}=$ $i \circ R_{J}$. Given $[a] \in H \widetilde{\Delta}(\widetilde{J})$, one has

$$
\begin{aligned}
\Psi(\widetilde{J} \widetilde{K}) \circ \widetilde{i}_{*}[a] & =\Psi(\widetilde{J} \widetilde{K})[\widetilde{i}(a)]=\left[R_{J K} \circ \widetilde{i}(a)\right] \\
& =\left[i \circ R_{J}(a)\right]=i_{*}\left[R_{J}(a)\right]=i_{*} \circ \Psi(\widetilde{J})[a] .
\end{aligned}
$$

- $\Psi(\widetilde{K}) \circ \widetilde{p}_{*}=p_{*} \circ \Psi(\widetilde{J} \widetilde{K})$.

Similarly, $\left.R_{J K}\right|_{C \widetilde{\Delta}(\widetilde{K})}=R_{K}$ and $R_{K} \circ \widetilde{p}=p \circ R_{J K}$. Given $[a] \in H \widetilde{\Delta}(\widetilde{J} \widetilde{K})$, one has

$$
\begin{aligned}
\Psi(\widetilde{K}) \circ \widetilde{p}_{*}[a] & =\Psi(\widetilde{K})[\widetilde{p}(a)]=\left[R_{K} \circ \widetilde{p}(a)\right] \\
& =\left[p \circ R_{J K}(a)\right]=p_{*}\left[R_{J K}(a)\right]=p_{*} \circ \Psi(\widetilde{J} \widetilde{K})[a] .
\end{aligned}
$$

Hence, we have shown that the diagram in (3.4) is commutative and, by our initial considerations, this suffice to prove that the graded module braid $\mathcal{H} \widetilde{\Delta}$ is isomorphic to the homology index braid $\mathcal{H}(\prec)$. This in turn, proves that the map $\widetilde{\Delta}: C \widetilde{P} \rightarrow C \widetilde{P}$ is a connection matrix of $\mathcal{H}(\prec)$.

\section{Application to Morse-Bott flows}

In this section, our goal is to obtain an explicit connection matrix of the finest Morse decomposition of a Morse-Bott flow in $M$. In order to do this, we will make use of the results in the previous section as well as a specific perturbation of $f$ described below.

To familiarize the reader with the main topic of this section, Morse Bott functions, we will present some basic definitions and results from Morse-Bott Theory. For more details about this subject, see [1] and [2].

Let $f: M \rightarrow \mathbb{R}$ be a smooth function on a smooth closed $n$-manifold. Suppose that the set of critical points of $f, \operatorname{Crit}(f)$, contains a closed $k$-submanifold $S$ of $M$. Choosing a Riemannian metric on $M$, the tangent space of $M$ restricted to $S$ splits as

$$
\left.T_{*} M\right|_{S}=T_{*} S \oplus \nu_{*} S,
$$

where $T_{*} S$ and $\nu_{*} S$ are the tangent and the normal bundles of $S$, respectively.

Let $\operatorname{Hess}_{p}(f)$ be the Hessian of $f$ at $p \in S \subset \operatorname{Crit}(f)$. Given $v \in T_{p} S$ and $w \in T_{p} M$, then

$$
\operatorname{Hess}_{p}(f)(v, w)=V_{p} \cdot(W \cdot f)=0,
$$

since $V_{p} \in T_{p} S$ and any extension of $w$ to a vector field $W$ satisfies $\left.d f(W)\right|_{S}=0$. Therefore, the Hessian $\operatorname{Hess}_{p}(f)$ induces a symmetric bilinear form on the normal space $\nu_{p} S$, denoted by $\operatorname{Hess}_{p}^{\nu}(f)$.

A smooth function $f: M \rightarrow \mathbb{R}$ on a closed manifold $M$ is called a Morse-Bott function if the set of critical points $\operatorname{Crit}(f)$ is a disjoint union of connected closed 
submanifolds of $M$, which are called critical manifolds of $f$, and for each critical manifold $S$, the bilinear form $\operatorname{Hess}_{p}^{\nu}(f)$ is non-degenerate for all $p \in S$. One says that the Hessian is non-degenerate in the normal direction to the critical manifolds.

Given $p \in \operatorname{Crit}(f)$, where $f$ is a Morse-Bott function, the Morse-Bott index of $p$ is defined to be the maximal dimension of a subspace of $\nu_{p} S$ on which $\operatorname{Hess}_{p}^{\nu}(f)$ is negative definite. The Morse-Bott index of a critical point $p$ will be denoted by $\lambda_{p}$.

For the following lemma see [1].

Lemma 4.1 (Morse-Bott Lemma). Let $f: M \rightarrow \mathbb{R}$ be a Morse-Bott function on a n-dimensional manifold $M$ and $S \subset \operatorname{Crit}(f)$ a critical manifold. For each $p \in S$, there exists a local chart $\phi$ of $M$ around $p$ and a local splitting of the normal bundle of $S, \nu_{*} S=\nu_{*}^{+} S \oplus \nu_{*}^{-} S$, which identifies a point $x$ in the domain of $\phi$ to $(u, v, w)$, where $u \in S, v \in \nu_{*}^{-} S$ and $w \in \nu_{*}^{+} S$, such that

$$
f \circ \phi^{-1}(u, v, w)=f(u)-|v|^{2}+|w|^{2} .
$$

Note that, by the Morse-Bott Lemma, if $S$ is a connected critical manifold then $\lambda_{p}$ is constant throughout $S$, that is, $\lambda_{p}=\lambda_{q}$, for all $p, q \in S$. Hence, one can refer to $\lambda_{p}$ as the Morse-Bott index $\lambda_{S}$ of the connected critical manifold $S$. Moreover, Lemma 4.1 shows that, at a critical point $p \in S$, the tangent space splits in the following way

$$
T_{p} M=T_{p} S \oplus \nu_{*}^{+} S \oplus \nu_{*}^{-} S,
$$

where $\lambda_{p}=\operatorname{dim}\left(\nu_{p}^{-} S\right)$. If $k=\operatorname{dim} S$ and $\lambda_{p}^{*}=\operatorname{dim}\left(\nu_{p}^{+} S\right)$, then one has the relation $n=k+\lambda_{p}+\lambda_{p}^{*}$.

Let $f: M \rightarrow \mathbb{R}$ be a Morse-Bott function on a smooth closed manifold $M$ of finite dimension $n$. In [1], a perturbation technique of Morse-Bott functions to Morse-Smale functions is presented. The perturbation defined therein produces an explicit Morse-Smale function $h: M \rightarrow \mathbb{R}$ which is arbitrarily close to a given Morse-Bott function $f$, such that, $h=f$ outside of a neighborhood of the critical set of $f$. More specifically, if $f$ has $l$ disjoint connected critical manifolds, namely $S_{1}, \ldots, S_{l}$, then $h$ is given by the expression

$$
h=f+\varepsilon\left(\sum_{j=1}^{l} \rho_{j} f_{j}\right)
$$

where $f_{j}$ is a Morse-Smale function on a tubular neighbourhood $T_{j}$ of the critical manifold $S_{j}$ and $\rho_{j}$ is a bump function which is identically 1 near $S_{j}$ and identically zero outside $T_{j}$, for each $j=1, \ldots, l$. The critical points of $h$ are exactly the union of the critical points of $f_{j}$, for all $j=1, \ldots, l$. Moreover, if $p$ is a critical point of $f_{j}$ of index $\lambda_{p}^{j}$, then $p$ is a critical point of $h$ of index 
$\lambda_{p}^{h}=\lambda_{j}+\lambda_{p}^{j}$, where $\lambda_{j}$ denotes the Morse-Bott index of the critical manifold $S_{j}$. For more details see [1].

Consider a flow $\varphi_{f}$ in $M$ generated by $f$, that is, a flow associated to the vector field $-\nabla f$. Throughout this article, flows generated by a Morse-Bott function $f$ will be call Morse-Bott flows. Let $h$ be a Morse-Smale perturbation of $f$, as described above, and denote by $\varphi_{h}$ a Morse-Smale flow associated to the vector field $-\nabla h$.

The next result provides a relation between the connection matrices for a Morse-Bott flow $\varphi_{f}$ and the connection matrices for a perturbed Morse-Smale flow $\varphi_{h}$ of $\varphi_{f}$. More specifically, we prove that the set of connection matrices of a Morse decomposition of $M$ relative to $\varphi_{f}$ is equal to the set of connection matrices of the induced coarser Morse decomposition of $M$ relative to $\varphi_{h}$.

Theorem 4.2. Let $f$ be a Morse-Bott function on $M$ and $h$ a Morse-Smale perturbation of $f$. If $\mathcal{D}(M)$ is a $\prec$-ordered Morse decomposition of $M$ relative to the flow $\varphi_{f}$, then $\mathcal{D}(M)$ is also a $\prec$-ordered Morse decomposition of $M$ relative to the flow $\varphi_{h}$ and the sets of connection matrices relative to the both flows are equal, i.e.

$$
\mathcal{C M}\left(\prec ; \varphi_{f}\right)=\mathcal{C M}\left(\prec ; \varphi_{h}\right) .
$$

Proof. Let $\left\{S_{1}, \ldots, S_{l}\right\}$ be the critical manifolds of $f$ and $N_{j}$ small isolating neighbourhoods of $S_{j}$, for each $j=1, \ldots, l$. Without loss of generality, one can consider $h$ as a perturbation of $f$ such that $f=h$ in $M \backslash N$, where $N=\bigcup_{j=1}^{l} N_{j}$. Then $\varphi_{f}$ and $\varphi_{h}$ coincide in $M \backslash N$, since $\nabla f=\nabla h$ in $M \backslash N$.

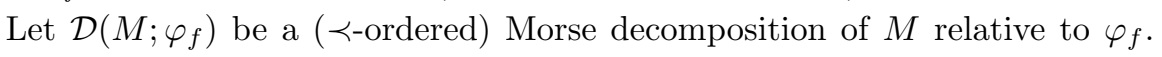
Since $\varphi_{f}$ and $\varphi_{h}$ coincide outside $N$, this set is also a Morse decomposition of $M$ relative to the perturbed flow $\varphi_{h}$, which will be denoted by $\widetilde{\mathcal{D}}\left(M ; \varphi_{h}\right)$. Moreover, an admissible ordering of $\mathcal{D}\left(M ; \varphi_{f}\right)$ is also an admissible ordering of $\widetilde{\mathcal{D}}\left(M ; \varphi_{h}\right)$ and conversely; flow orderings of both Morse decompositions coincide. Therefore, for each interval $J$ of $\prec$, the Conley index of $M_{J}$ as Morse set of $\mathcal{D}\left(M ; \varphi_{f}\right)$ is equal to the Conley index of $M_{J}$ as Morse set of $\widetilde{\mathcal{D}}\left(M ; \varphi_{h}\right)$. Furthermore, the homology index braid of the admissible ordering $\prec$ of $\mathcal{D}\left(M ; \varphi_{f}\right)$ coincides with the homology index braid of the admissible ordering $\prec$ of $\widetilde{\mathcal{D}}\left(M ; \varphi_{h}\right)$. Hence, the collection $\mathcal{C M}\left(\prec ; \varphi_{f}\right)$ of connection matrices of the admissible ordering $\prec$ of $\mathcal{D}\left(M ; \varphi_{f}\right)$ is equal to the collection $\mathcal{C M}\left(\prec ; \varphi_{h}\right)$ of connection matrices of the admissible ordering $\prec$ of $\widetilde{\mathcal{D}}\left(M ; \varphi_{h}\right)$.

Note that the set $\left\{S_{1}, \ldots, S_{l}\right\}$ of all critical manifolds of $f$ is a Morse decomposition of $M$ with respect to the flow $\varphi_{f}$. Denote this Morse decomposition, which is the finest one, by $\mathcal{D}\left(M ; \varphi_{f}\right)$. On the other hand, if $h$ is a Morse-Smale perturbation of $f$, denote the finest Morse decomposition of $M$ relative to $\varphi_{h}$ 
by $\mathcal{D}\left(M ; \varphi_{h}\right)$. The next theorem provides a relation between the connections matrices of both Morse decompositions. More specifically, we prove that each connection matrix of $\mathcal{D}\left(M ; \varphi_{h}\right)$ induces a connection matrix of $\mathcal{D}\left(M ; \varphi_{f}\right)$.

For the next result, the homology is computed over $\mathbb{Z}$ or over a field.

Theorem 4.3. Let $f$ be a Morse-Bott function on $M$ and $h$ be a MorseSmale perturbation of $f$ such that $h$ restricted to a critical manifold of $f$ is a perfect Morse function. Then a connection matrix of $\mathcal{D}\left(M ; \varphi_{h}\right)$ induces a connection matrix of $\mathcal{D}\left(M ; \varphi_{f}\right)$.

Proof. By Theorem 4.2, the Morse decomposition $\mathcal{D}\left(M ; \varphi_{f}\right)=\left\{S_{1}, \ldots, S_{l}\right\}$ is also a Morse decomposition of $M$ relative to $\varphi_{h}$ and it will be denoted by $\widetilde{\mathcal{D}}\left(M ; \varphi_{h}\right)$. Both Morse decompositions are $\prec_{F}$-ordered, where $\prec_{F}$ is the admissible flow ordering. Observe that $\mathcal{D}\left(M ; \varphi_{f}\right)$ is the finest Morse decomposition of $M$ in $\varphi_{f}$, but it is not the case of $\widetilde{\mathcal{D}}\left(M ; \varphi_{h}\right)$. Denote by $\mathcal{D}\left(M ; \varphi_{h}\right)$ the finest Morse decomposition of $M$ relative to $\varphi_{h}$, that is, each critical point of $h$ corresponds to a Morse set of $\mathcal{D}\left(M ; \varphi_{h}\right)$.

Now, using the main result of Section 3, we will induce a connection matrix of $\widetilde{\mathcal{D}}\left(M ; \varphi_{h}\right)$ from $\mathcal{D}\left(M ; \varphi_{h}\right)$. Since, by Theorem $4.2, \mathcal{C} \mathcal{M}\left(\prec_{F} ; \varphi_{f}\right)=\mathcal{C M}\left(\prec_{F} ; \varphi_{h}\right)$, we will obtain the required connection matrix of $\mathcal{D}\left(M ; \varphi_{f}\right)$.

Observe that the finest Morse decomposition $\mathcal{D}\left(M ; \varphi_{h}\right)=\left\{M_{\pi}\right\}_{\pi \in P}$ of $M$ is $<_{F}$-ordered, where $<_{F}$ denotes the flow ordering. Considering the intervals $I_{1}, \ldots, I_{l}$, where $I_{j}=\left\{\pi \in P: M_{\pi} \in S_{j}\right\}$, then $\widetilde{\mathcal{D}}\left(M ; \varphi_{h}\right)$ is obtained from $\mathcal{D}\left(M ; \varphi_{h}\right)$ by defining $\widetilde{M}_{\{\pi\}}=M_{\pi}$, if $\pi \notin I_{1}, \ldots, I_{l}$ and $\widetilde{M}_{I_{j}}=M_{I_{j}}$, for $j=$ $1, \ldots, l$. Given a connection matrix $\Delta$ of $\mathcal{D}\left(M ; \varphi_{h}\right)$, observe that each submatrix $\Delta\left(I_{j}\right)$, for $j=1, \ldots, l$, corresponds to a connection matrix of the finest Morse decomposition of $S_{j}$ under the flow restricted to $S_{j}$, since each non null map in $\Delta\left(I_{j}\right)$ is flow defined. By hypothesis, $\left.h\right|_{S_{j}}$ is a perfect Morse function. Hence, by Proposition 2.1, one has that $\Delta\left(I_{j}\right)=0$, for all $j$. Therefore, we are able to apply Theorem 3.7 which provides a connection matrix $\widetilde{\Delta}$ of $\widetilde{\mathcal{D}}\left(M ; \varphi_{h}\right)$.

EXAMPLE 4.4. Let $f$ be a Morse-Bott function on $S^{2}$ having three isolated critical points of indices 2, namely $x_{1}, x_{2}$ and $x_{3}$, one isolated critical point of index 1, namely, $y$ and $B=S^{1}$ as critical manifold of index 0 , as depicted in Figure 1.

Let $h$ be a perturbation of $f$ when one considers a perfect Morse function on the critical manifold $S^{1}$, as in Figure 2. Denote by $\varphi_{f}$ (resp. $\varphi_{h}$ ) a Morse-Bott flow (resp. Morse flow) on $S^{2}$ associated to the vector field $-\nabla f$ (resp. $-\nabla h$ ).

The objective herein is to obtain a connection matrix of the finest Morse decomposition of $S^{2}$ with respect to the Morse-Bott flow $\varphi_{f}$ by means of a connection matrix of the finest Morse decomposition of $S^{2}$ with respect to the Morse flow $\varphi_{h}$. Firstly, we will compute a connection matrix of a Morse decomposition 


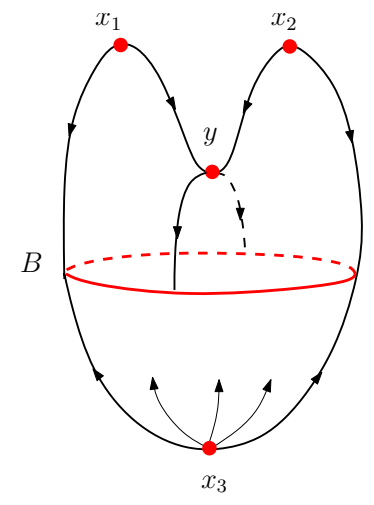

Figure 1. A Morse-Bott flow on $S^{2}$ associated to the function $f$

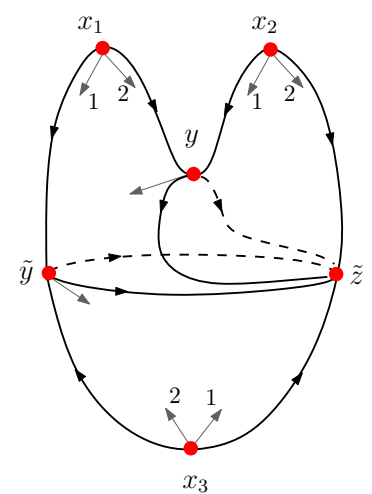

Figure 2. A Morse-Smale perturbation of the Morse-Bott function $f$

of $S^{2}$ with respect to $\varphi_{h}$. Then, using the tools proved in Section 3, we will obtain the required matrix.

Consider the set $P=\{1,2,3,4,5,6\}$ with partial order $<$ given by $[1<2,3]$; $[2<4,5]$; and $[1<4,6]$. Let $M_{1}=\widetilde{z}, M_{2}=\tilde{y}, M_{3}=y, M_{4}=x_{1}, M_{5}=x_{2}$ and $M_{6}=x_{3}$. The set $\mathcal{D}\left(S^{2} ; \varphi_{h}\right)=\left\{M_{i}: i \in P\right\}$ is the finest <-ordered Morse decomposition of $S^{2}$ with respect to the flow $\varphi_{h}$. Moreover, $<$ is the flow ordering. As proved by Salamon in [14], the differential of a Morse-Witten complex of $h$ is a connection matrix of the Morse decomposition $\mathcal{D}\left(S^{2} ; \varphi_{h}\right)$. In order to obtain a connection matrix of $\mathcal{D}\left(S^{2} ; \varphi_{h}\right)$, we will compute the MorseWitten complex $\left(C_{*}(h), \partial_{*}\right)$ of $h$, considering the orientations on the unstable manifolds of the critical points as the ones illustrated in Figure 2. For this choice of orientations, we have that the Morse chain groups are $C_{0}(f)=\mathbb{Z}\langle\widetilde{z}\rangle$, $C_{1}(f)=\mathbb{Z}\langle\widetilde{y}\rangle \oplus \mathbb{Z}\langle y\rangle$ and $C_{2}(f)=\mathbb{Z}\left\langle x_{1}\right\rangle \oplus \mathbb{Z}\left\langle x_{2}\right\rangle \oplus \mathbb{Z}\left\langle x_{3}\right\rangle$, where $\langle x\rangle$ denotes both the critical point $x$ as well as its orientation. The differential $\partial_{*}^{c}$ is defined 
on the generators according to the matrix:

$$
\partial_{*}=\begin{array}{r}
\widetilde{z} \\
\widetilde{z} \\
\widetilde{y} \\
y \\
x_{1} \\
x_{2} \\
x_{3}
\end{array}\left[\begin{array}{cccccc}
0 & 0 & 0 & 0 & 0 & 0 \\
0 & 0 & 0 & +1 & 0 & -1 \\
0 & 0 & 0 & -1 & +1 & 0 \\
0 & 0 & 0 & 0 & 0 & 0 \\
0 & 0 & 0 & 0 & 0 & 0 \\
0 & 0 & 0 & 0 & 0 & 0
\end{array}\right],
$$

which is a connection matrix of $\mathcal{D}\left(S^{2}, \varphi_{h}\right)$ by identifying the number \pm 1 with an isomorphism between the Conley homology indices in question.

Now, note that $I=\{1,2\}$ is an interval in $(P,<)$ and, defining $\widetilde{P}=\{I,\{3\}$, $\{4\},\{5\},\{6\}\}$ and the order $[I \prec\{3\} \prec\{4\},\{5\}] ;[I \prec\{6\}]$, then $(\widetilde{P}, \prec)$ is a partially ordered set, by Proposition 3.1. Let $\widetilde{\mathcal{D}}\left(S^{2} ; \varphi_{h}\right)=\left\{\widetilde{M}_{\widetilde{\pi}}: \widetilde{\pi} \in \widetilde{P}\right\}$, where $\widetilde{M}_{I}=\tilde{y} \cup C\left(M_{2}, M_{1}\right) \cup \tilde{z}$ and $\widetilde{M}_{\{i\}}=M_{i}$, for $i \in\{3,4,5,6\}$. By Proposition 3.3, this set is an $\prec$-ordered Morse decomposition of $S^{2}$ with respect to the flow $\varphi_{h}$. Moreover, by Proposition 3.4, $\prec$ is the flow ordering. Finally, by Theorem 3.7, $\Delta$ induces a connection matrix $\widetilde{\Delta}$ of $\widetilde{\mathcal{D}}\left(S^{2}, \varphi_{h}\right)$, which is given by the following map of degree -1 from $C H(B) \oplus C H(y) \oplus C H\left(x_{1}\right) \oplus C H\left(x_{2}\right) \oplus C H\left(x_{3}\right)$ to itself:

$$
\widetilde{\Delta}=\begin{array}{rllll}
B & y & x_{1} & x_{2} & x_{3} \\
B & y \\
x_{1} \\
x_{2} \\
x_{3}
\end{array}\left[\begin{array}{ccccc}
0 & 0 & \approx & 0 & \approx \\
0 & 0 & \approx & \approx & 0 \\
0 & 0 & 0 & 0 & 0 \\
0 & 0 & 0 & 0 & 0 \\
0 & 0 & 0 & 0 & 0
\end{array}\right] .
$$

Consider the $\prec$-ordered Morse decomposition $\widetilde{\mathcal{D}}\left(S^{2} ; \varphi_{f}\right)=\left\{M_{\widetilde{\pi}}: \widetilde{\pi} \in \widetilde{P}\right\}$ relative to the Morse-Bott flow $\varphi_{f}$, where $M_{I}=B, M_{\{3\}}=y, M_{\{4\}}=x_{1}$, $M_{\{5\}}=x_{2}$ and $M_{\{6\}}=x_{3}$. The partial order $\prec$ is the flow ordering. By Theorem 4.3, the map in (4.1) is a connection matrix of $\mathcal{D}\left(S^{2} ; \varphi_{f}\right)$.

In the previous example, one could have chosen a different perturbation $\tilde{h}$ of $f$. For instance, using the perturbation shown in Figure 3, one obtains the following map from $C H(B) \oplus C H(y) \oplus C H\left(x_{1}\right) \oplus C H\left(x_{2}\right) \oplus C H\left(x_{3}\right)$ to itself:

$$
\Delta=\begin{array}{rllll}
B & y & x_{1} & x_{2} & x_{3} \\
B \\
y
\end{array}\left[\begin{array}{ccccc}
0 & 0 & 0 & \approx & \approx \\
0 & 0 & \approx & \approx & 0 \\
x_{1} \\
x_{2} \\
x_{3}
\end{array}\left[\begin{array}{ccccc}
0 & 0 & 0 & 0 & 0 \\
0 & 0 & 0 & 0 & 0 \\
0 & 0 & 0 & 0 & 0
\end{array}\right],\right.
$$


which is a connection matrix of the finest Morse decomposition of $S^{2}$ relative to the flow $\varphi_{f}$. Therefore, we do not have the uniqueness of connection matrices in Morse-Bott flows, even if the homology were computed over a field.

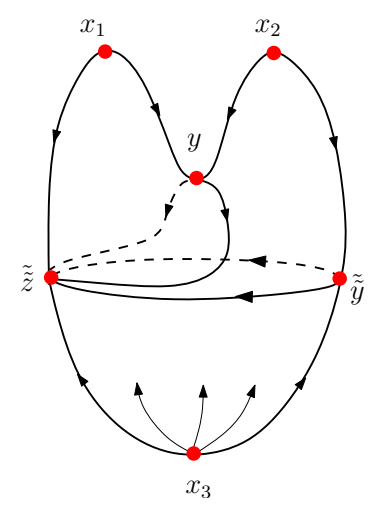

Figure 3. A Morse perturbation of the Morse-Bott function $f$

\section{Final remarks}

At this point, a natural question arises: are all connection matrices of the finest Morse decomposition of a Morse-Bott flow $\varphi_{f}$ obtained via Theorem 4.3? In other words, for each connection matrix $\Delta$ of the finest Morse decomposition of a Morse-Bott flow is there a Morse-Smale perturbation $\varphi_{h}$ of $\varphi_{f}$ such that $\Delta$ is induced from a connection matrix of $\varphi_{h}$ ?

In general, this is not the case as seen in Example 5.2. On the other hand, if some additional structure is assumed it may hold true, as can be verified in Examples 4.4 and 5.1.

ExAmple 5.1. Consider $S^{3}$ as the manifold obtained from gluing two solid tori $\mathcal{T}_{1}$ and $\mathcal{T}_{2}$ by a homeomorphism of their boundaries, the tori $T_{1}$ and $T_{2}$, which identifies a parallel of $T_{1}$ to the meridian of $T_{2}$. Let $f$ be a Morse-Bott function on $S^{3}$ such that the critical manifolds of $f$ are the torus $T_{1}$ as a repeller and two 1-spheres $S_{1}$ and $S_{2}$ as attractors, where $S_{i}$ lies in the interior of $\mathcal{T}_{i}$, $i=1,2$. Their Morse-Bott indices are $\lambda_{T_{1}}=1, \lambda_{S_{1}}=0$ and $\lambda_{S_{2}}=0$.

Considering the finest Morse decomposition $\mathcal{D}\left(S^{3}\right)=\left\{S_{1}, S_{2}, T_{1}\right\}$ of $S^{3}$ with the admissible flow ordering and a connection matrix $\widetilde{\Delta}$ of $\mathcal{D}\left(S^{3}\right)$, then the only possible non null maps are $\widetilde{\Delta}\left(T_{1}, S_{1}\right)$ and $\widetilde{\Delta}\left(T_{1}, S_{2}\right)$, which are flow defined. Hence, there is a unique connection matrix of $\mathcal{D}\left(S^{3}\right)$, namely,

$$
\widetilde{\Delta}=\begin{gathered}
S_{1} \\
S_{1} \\
S_{2} \\
T_{1}
\end{gathered}\left[\begin{array}{ccc}
0 & 0 & T_{1} \\
0 & 0 & \widetilde{\Delta}_{2} \\
0 & 0 & 0
\end{array}\right] .
$$


We will show that this map can be obtained from a connection matrix of a perturbation of the function $f$, as discussed in Section 4 . In this sense, let $h$ be a Morse-Smale perturbation of $f$, such that it is a perfect Morse function when restricted to the the critical manifolds, $S_{1}, S_{2}, T_{1}$, of $f$. Denote the critical points of $h$ by $z_{1}, y_{1} \in S_{1}, z_{2}, y_{2} \in S_{2}$ and $x, v_{1}, v_{2}, y_{3} \in T_{1}$, where $z_{i}^{\prime} s$ have indices zero, $y^{\prime} s$ have indices one, $v^{\prime} s$ have indices two and $x$ has index three. The differential of the Morse complex $\left(C_{*}(f), \partial_{*}^{c}\right)$ over $\mathbb{Z}_{2}$ associated to $h$ is given by the matrix:

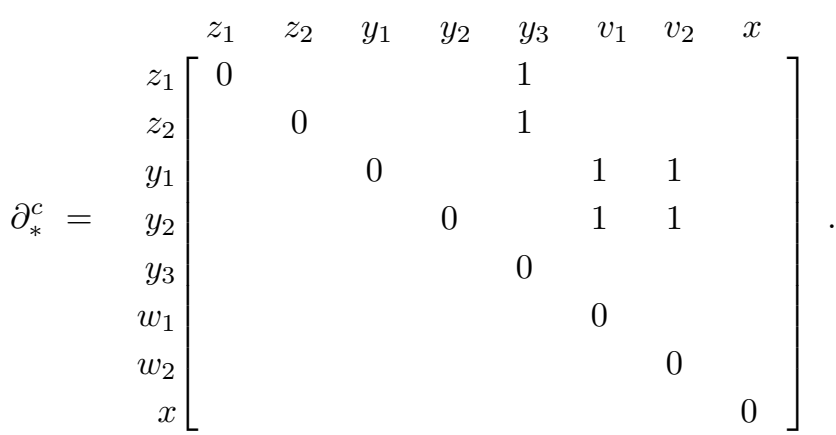

Note that the Conley homological indices of the critical manifolds are as follows:

$$
C H_{n}\left(T_{1}\right)=\left\{\begin{array}{ll}
\mathbb{Z}_{2} & \text { for } n=1,3, \\
\mathbb{Z}_{2} \oplus \mathbb{Z}_{2} & \text { for } n=2, \\
0 & \text { for } n \neq 1,2,3,
\end{array} \quad C H_{n}\left(S_{i}\right)= \begin{cases}\mathbb{Z} & \text { for } n=0,1 \\
0 & \text { for } n \neq 0,1\end{cases}\right.
$$

where $i=1,2$. Hence, there exist isomorphisms $C H\left(S_{i}\right) \cong C H\left(z_{i}\right) \oplus C H\left(y_{i}\right)$, for $i=1,2$, and $C H\left(T_{1}\right) \cong C H\left(y_{3}\right) \oplus C H\left(v_{1}\right) \oplus C H\left(v_{2}\right) \oplus C H(x)$, which will be denoted by $F_{i}$ and $F_{T_{1}}$, respectively. Now, observe that the submatrix of $\partial_{*}^{c}$

$$
\Delta_{i}=z_{i}\left[\begin{array}{llll}
y_{3} & v_{1} & v_{2} & x \\
1 & 0 & 0 & 0 \\
0 & 1 & 1 & 0
\end{array}\right]
$$

induces a map $\widetilde{\Delta}_{i}: C H\left(T_{1}\right) \rightarrow C H\left(S_{i}\right)$, for $i=1,2$, by composing $\Delta_{i}$ with the isomorphisms $F_{T_{1}}$ and $F_{i}^{-1}$. Therefore, the induced connection matrix of the finest Morse decomposition of $S^{3}$ relative to the Morse-Bott flow $\varphi_{f}$ is

$$
\widetilde{\Delta}=\begin{gathered}
S_{1} \\
S_{1} \\
S_{2} \\
T_{1}
\end{gathered}\left[\begin{array}{ccc}
0 & 0 & T_{1} \\
0 & 0 & \widetilde{\Delta}_{1} \\
0 & 0 & 0
\end{array}\right]
$$

The following is an adaptation to our context of Reineck's example in [13] and illustrates that there are connection matrices that do not arise from MorseSmale perturbation. 
ExAmPle 5.2. Consider a flow in $\mathbb{R}^{2}$ as in Figure 4 having seven singularities and one critical manifold diffeomorphic to $S^{1}$. By taking the one point compactification $\mathbb{R}^{2} \cup\{\infty\}$ of $\mathbb{R}^{2}$ and letting $\infty$ be an attractor point, one obtains a Morse-Bott flow on $M=S^{2}$.

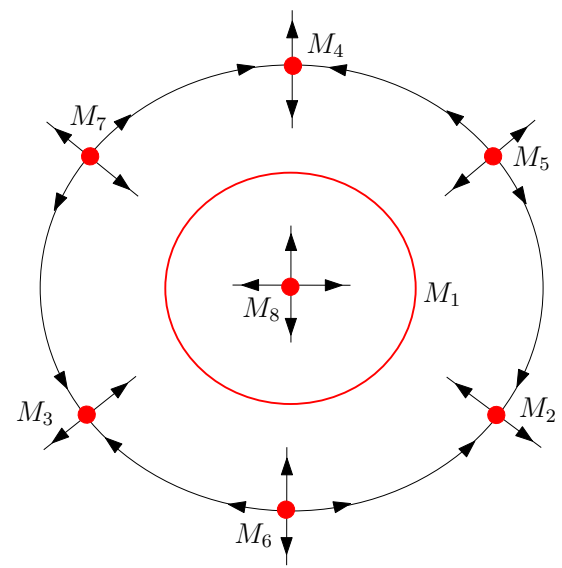

Figure 4. Morse-Bott flow on $\mathbb{R}^{2}$

The critical manifold together with the eight isolated singularities form a Morse decomposition $\mathcal{D}\left(S^{2}\right)$ of $M$, with flow ordering [0<2,3,4]; $[1<2,3,4,8]$; $[2<5,6] ;[3<6,7]$ and $[4<5,7]$, in $P=\{1, \ldots, 8\}$. Let $\Delta$ be a connection matrix of $\mathcal{D}\left(S^{2}\right)$. All maps $\Delta\left(\pi^{\prime}, \pi\right)$ are flow defined, except for $\Delta(5,1), \Delta(6,1)$ and $\Delta(7,1)$. It is easy to compute the boundary flow defined maps using index triples. In this example they are all isomorphism. The connection matrix $\Delta$ is as follows:

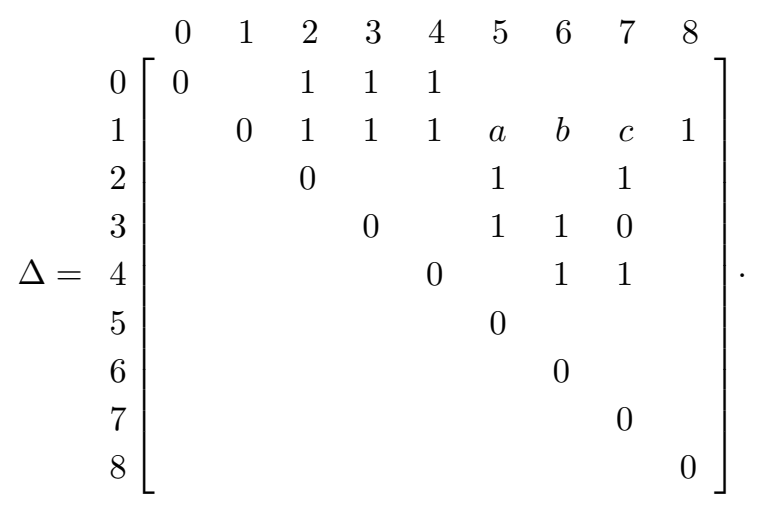

The homology with $\mathbb{Z}_{2}$-coefficients was used in order to simplify computations, however one could as well have used $\mathbb{Z}$-coefficients. 
The maps $a, b$ and $c$ in $\Delta$ are not flow defined. As $\Delta$ is a connection matrix, then

$$
\frac{\operatorname{Ker} \Delta(P)}{\operatorname{Im} \Delta(P)}=H \Delta(P) \cong C H(M)=H_{*}\left(M ; \mathbb{Z}_{2}\right)= \begin{cases}\mathbb{Z}_{2} & \text { for } n=2,0 \\ 0 & \text { c.c. }\end{cases}
$$

which implies that:

- $\frac{\operatorname{Ker} \Delta_{0}}{\operatorname{Im} \Delta_{1}} \cong \mathbb{Z}_{2}$, which implies that the rank of $\Delta_{1}$ must be 1 ;

- $\frac{\operatorname{Ker} \Delta_{1}}{\operatorname{Im} \Delta_{2}} \cong 0$, which implies that the rank of $\Delta_{2}$ must be equal to 3 ;

- $\frac{\operatorname{Ker} \Delta_{2}}{\operatorname{Im} \Delta_{3}} \cong \mathbb{Z}_{2}$, which implies that the rank of the kernel of $\Delta_{2}$ must be equal to 1 .

By the last item, we must have $a+b+c \neq 0(\bmod 2)$. In other orders, either one of these entries is one or all of these entries are one. Therefore, combining possibilities, one has four connection matrices of $\mathcal{D}(M)$. Three of these connection matrices are obtained from connection matrices of a Morse-Smale perturbation, namely the ones where only one of the entries $a, b, c$ is one. On the other hand, the connection matrix where $a=b=c=1$ can not be obtained from a Morse flow by substituting the critical manifolds for two singularities, since the saddle has only two orbits in its stable manifold.

Therefore not all connection matrices for Morse-Bott flows arise from connection matrices for Morse-Smale perturbations.

\section{REFERENCES}

[1] A. Banyaga And D. Hurtubise, The Morse-Bott inequalities via a dynamical systems approach, Ergodic Theory Dynam. Systems 29 (2009), no. 6, 1693-1703.

[2] _ Lecture on Morse Homology, Kluwer Texts in the Mathematical Sciences, vol. 29, Kluwer Academic Publishers Group, Dordrecht, (2004).

[3] C. Conley, Isolated Invariant Sets and the Morse Index, CBMS Regional Conference Series in Math. AMS, Providence, RI 38 (1978).

[4] O. Cornea, K.A. De Rezende And M.R. DA Silveira, Spectral sequences in Conley's theory, Ergodic Theory Dynam. Systems 30 (4) (2010), 1009-1054 .

[5] R. Franzosa, Index filtrations and the homology index braid for partially ordered Morse fecompositions, Trans. Amer. Math. Soc. 298 (1986), 193-213.

[6] _ The connection matrix theory for Morse decompositions, Trans. Amer. Math. Soc. 311 (1989), 561-592.

[7] _ , The continuation theory for Morse decompositions and connection matrices, Trans. Amer. Math. Soc. 310 (1988), 781-803.

[8] R. Franzosa, K.A. De Rezende and M.R. DA Silveira, Continuation and bifurcation associated to the dynamical spectral sequence, Ergodic Theory Dynam. Systems, available on CJO 2013 doi:10.1017/etds.2013.29.

[9] D. Lima And K. DE Rezende, Generalized Morse-Bott Inequalities, preprint. 
[10] C. MCCORD AND K. Mischaikow, Connected simple systems, transition matrices and heteroclinic bifurcations, Trans. Amer. Math. Soc. 333 (1992), 397-422.

[11] M.P. Mello, K.A. De Rezende And M.R. DA Silveira, Conley's spectral sequences via the sweeping algorithm, Topology Appl. 157 (13) (2010), 2111-2130.

[12] J. REINECK, Connecting orbits in one-parameter families of flows, Ergodic Theory Dynam. Systems 8 (1988), 359-374.

[13] - The connection matrix in Morse-Smale flows, Trans. Amer. Math. Soc. 322 (1990), 523-545.

[14] D.A. Salamon, The Morse theory, the Conley index and the Floer homology, Bull. London Math. Soc. 22 (1990), 113-240.

[15] J. WeBer, The Morse-Witten complex via dynamical systems, Expo. Math. 24 (2006), $127-159$.

Manuscript received October 2, 2013

Dahisy V. De S. Lima And Ketty A. DE Rezende

Departamento de Matemática

Universidade Estadual de Campinas

13.083-85 Campinas - SP, BRAZIL

TMNA : Volume $44-2014-\mathrm{N}^{\mathrm{O}} 2$ 